\title{
Sparse learning of stochastic dynamical equations
}

\author{
Lorenzo Boninsegna, Feliks Nüske, and Cecilia Clementi \\ Department of Chemistry and Center for Theoretical Biological Physics, Rice University, \\ Houston, Texas 77005, USA
}

(Received 6 December 2017; accepted 8 March 2018; published online 30 March 2018)

\begin{abstract}
With the rapid increase of available data for complex systems, there is great interest in the extraction of physically relevant information from massive datasets. Recently, a framework called Sparse Identification of Nonlinear Dynamics (SINDy) has been introduced to identify the governing equations of dynamical systems from simulation data. In this study, we extend SINDy to stochastic dynamical systems which are frequently used to model biophysical processes. We prove the asymptotic correctness of stochastic SINDy in the infinite data limit, both in the original and projected variables. We discuss algorithms to solve the sparse regression problem arising from the practical implementation of SINDy and show that cross validation is an essential tool to determine the right level of sparsity. We demonstrate the proposed methodology on two test systems, namely, the diffusion in a onedimensional potential and the projected dynamics of a two-dimensional diffusion process. Published by AIP Publishing. https://doi.org/10.1063/1.5018409
\end{abstract}

\section{INTRODUCTION}

The last decade has seen a dramatic increase in our ability to collect or produce large amounts of high resolution and high dimensional data associated with complex physical and chemical systems, both by means of experimental measurements or computer simulations. In many different scientific fields, ranging from high energy physics to neuroscience, the "big-data" problem has spurred interest in data analysis methods that can condense massive datasets into a minimal amount of essential information and/or can detect relevant patterns and anomalies in the distribution of the data.

In the specific case of molecular systems, a large body of work has been devoted to defining collective coordinates and reaction pathways from molecular dynamics simulation data. ${ }^{1,2}$ However, most of the proposed techniques are descriptive and do not provide a functional link relating the variables to the observed behavior. Mathematical approaches that have been proved to be optimal to reduce the complexity of the data by dimensionality reduction and/or coarse graining (in time or space) usually do not offer a straightforward physical interpretation of the results. Here we take a different approach and make a first step toward the definition of methods to learn the functional form of a molecular model from the available data.

Assuming an extensive sampling of a given set of variables describing a system is available for a certain time frame, different data-driven methods have been proposed to "learn" how to propagate the system to future times, either in terms of the original variables or in a reduced representation. For instance, the so-called "equation-free" approach uses local (in time and space) microscopic simulations to propagate macroscopic variables to long time scales. ${ }^{3}$ Such an approach bypasses the need of formulating constitutive equations for the time evolution of the macroscopic variables of the system in closed form and provides a practical recipe for multiscale simulation. However, it is oftentimes desirable to obtain an explicit analytical expression for the dynamical equations in terms of the variables of interest, as they can offer a physicochemical understanding of the system. Ideally, one would like to design approaches that are able to infer such equations from the available data. Recently, a significant step in this direction has been proposed for deterministic dynamical systems. ${ }^{4}$ The Sparse Identification of Nonlinear Dynamics (SINDy) approach combines ideas from sparse regression ${ }^{5,6}$ and compressed sensing ${ }^{7,8}$ to automatically discover the terms of the differential equations (either ordinary ${ }^{4}$ or partial $^{9}$ ) that best represent large sets of time-dependent data, given a suitable function library (as it will be discussed below). For instance, it was shown that SINDy can be used to obtain the correct equation for the low-dimensional slow attractor associated with the dynamics of a fluid flow past a cylinder that is described by the Navier-Stokes equations at the microscopic scale. $^{4}$

Such a methodology appears to be very promising to learn effective equations of motion in different fields of application, such as molecular systems. A significant difference that limits the application of SINDy to (macro)molecular systems is the presence of noise, as their dynamics are usually non-deterministic. Toward this goal, here we present an extension of this approach that allows us to derive stochastic dynamical equations from data, to describe the time evolution either of microscopic variables or of their transformation in a different space. For the latter, we combine the SINDy idea with the formalism of projected stochastic dynamics. ${ }^{10,11}$ We show that extensive cross validation is a crucial ingredient that needs to be added in the sparsification of the solution for this approach to be successful in the presence of significant noise and/or limited data. Sparsity enforcement is a crucial step of the learning procedure since it results in parsimonious models with few descriptors which balance accuracy with complexity while avoiding overfitting; thus, 
efficiently implementing Occam's razor in the models being investigated.

The manuscript is organized as follows. First, the proposed extension of SINDy to stochastic systems and its theoretical underpinnings are outlined. We explain how trajectory data can be used as an input to formulate a regression problem approximating the drift and diffusion coefficients of an Ito process, both in the microscopic and in an effective variable space. The specific algorithm used to solve the regression is then detailed, by introducing a cross validation (CV) based Stepwise Sparse Regression. Such a formalism is employed to learn dynamical equations from data for two test systems: the homogeneous diffusion in a one dimensional double well potential and the projected dynamics along a projected coordinate in a two dimensional potential. Results and implications are finally discussed.

\section{THEORY}

\section{A. Sparse identification of dynamical systems}

We start by outlining the SINDy approach for deterministic dynamical systems that was originally proposed in Ref. 4. The goal is to learn the dynamical equations for a system described by an ordinary differential equation,

$$
\frac{d}{d t} X(t)=F(X(t)),
$$

where $X(t) \in \mathbb{R}^{d}$ is the state of the system at time $t$ and $F$ : $\mathbb{R}^{d} \rightarrow \mathbb{R}^{d}$ is the vector field defining the dynamics. For many complex systems, no closed-form expression for the vector field $F$ is known and the process can only be observed through simulation or measurement data $X\left(t_{l}\right), l=1, \ldots, N$, where $t_{1}<\cdots<t_{N}$ are discrete points in time. However, it was suggested in Ref. 4 to learn the dynamical equation as a linear combination of a pre-selected dictionary of basis functions. More precisely, let $\Theta_{K}=\left(f_{1}, \ldots, f_{K}\right)$ be a set of $K$ user-defined trial functions. Making the ansatz,

$$
F_{i}=\sum_{k=1}^{K} c_{i, k} f_{k}
$$

for the $i$ th component of the vector field, one arrives at a system of $N$ linear equations for each time step by inserting Eq. (2) into Eq. (1)

$$
\frac{d}{d t} X_{i}\left(t_{l}\right)=\sum_{k=1}^{K} c_{i, k} f_{k}\left(X\left(t_{l}\right)\right)
$$

If the time derivatives on the left hand side of Eq. (3) can be computed, this defines a linear system

$$
\mathbb{Y}_{i}=\mathbb{X} \mathbf{c}_{i}
$$

where $\mathbb{Y}_{i} \in \mathbb{R}^{N}$ contains the time derivatives at all sampled time steps, $\mathbb{X} \in \mathbb{R}^{N \times K}$ contains the evaluations of all basis functions in dictionary $\Theta_{K}$ at all time steps and $\mathbf{c}_{i} \in \mathbb{R}^{K}$ is the unknown vector of coefficients

$$
\begin{aligned}
Y_{i, l} & =\frac{d}{d t} X_{i}\left(t_{l}\right), \\
X_{l, k} & =f_{k}\left(X\left(t_{l}\right)\right) .
\end{aligned}
$$

Equation (4) needs to be solved in the least-squares sense, that is, $\tilde{\mathbf{c}}_{i}$ becomes the minimizer of

$$
\tilde{\mathbf{c}}_{i}=\underset{\mathbf{c}_{i} \in \mathbb{R}^{K}}{\arg \min }\left\|\mathbb{Y}_{i}-\mathbb{X} \cdot \mathbf{c}_{i}\right\|_{2}^{2} .
$$

In general, the solution $\tilde{\mathbf{c}}_{i}$ of Eq. (5) will not be sparse. If the goal is to find the minimal functional form of the vector field that reliably represents the data among the large number of possibilities offered in the function dictionary, sparsity of $\tilde{\mathbf{c}}_{i}$ needs to be enforced. Formally, this can be achieved by penalizing the $L^{1}$-norm of the solution and minimizing

$$
\tilde{\mathbf{c}}_{i}=\underset{\mathbf{c}_{i} \in \mathbb{R}^{K}}{\arg \min }\left(\left\|\mathbb{Y}_{i}-\mathbb{X} \mathbf{c}_{i}\right\|_{2}^{2}+\rho\left\|\mathbf{c}_{i}\right\|_{1}\right),
$$

using some positive Lagrange multiplier $\rho$ which controls the weight of the sparsity constraint. Algorithms to solve Eq. (6) will be discussed below.

\section{B. Sparse identification of stochastic dynamics}

\section{Diffusion processes}

In this work, we extend the sparse learning framework discussed above to stochastic dynamics. Instead of Eq. (1), we consider dynamics driven by an Ito stochastic differential equation (SDE)

$$
d X(t)=b(X(t)) d t+\sqrt{2 \beta^{-1}} \sigma(X(t)) d W_{t} .
$$

Again, $X(t) \in \mathbb{R}^{d}$ denotes the state of the system at time $t$, while $b: \mathbb{R}^{d} \mapsto \mathbb{R}^{d}$ is a vector field called the drift, and $\sigma: \mathbb{R}^{d} \mapsto \mathbb{R}^{d \times d}$ is a matrix field called the diffusion of the process. Moreover, $W_{t}$ denotes a $d$-dimensional Brownian motion and the prefactor $\beta$ is the inverse temperature $\beta=\frac{1}{k_{B} T}$ in physical applications. The covariance matrix of the diffusion is commonly denoted by $a \in \mathbb{R}^{d \times d}$,

$$
a(x)=\sigma^{T}(x) \sigma(x) .
$$

We will also refer to a process like Eq. (7) as a diffusion process. We assume the process $X(t)$ to be ergodic with respect to a unique invariant measure $\mu$. Although it is not strictly necessary for all of what follows, we also assume $X(t)$ to be reversible with respect to the invariant measure $\mu$.

A familiar example of such a process is the overdamped Langevin dynamics

$$
d X(t)=-\frac{\nabla U(X(t))}{\gamma} d t+\sqrt{2 \beta^{-1} \gamma} d W_{t},
$$

that is, the drift is the gradient of a potential energy function $U$ (the force) normalized by the friction coefficient $\gamma$, while the diffusion matrix is constant. The equilibrium distribution associated with this dynamical process is the Boltzmann distribution, $\mu(x) \propto \exp (-\beta U(x))$.

A diffusion process is thus generally defined by two components, the drift and the diffusion. Both of them can be estimated from data via the Kramers-Moyal formulae ${ }^{12}$

$$
b_{i}(x)=\lim _{s \rightarrow 0} \mathbb{E}\left[\frac{1}{s}\left(X_{i}(s)-x_{i}\right) \mid X(0)=x\right],
$$




$$
a_{i j}(x)=\frac{\beta}{2} \lim _{s \rightarrow 0} \mathbb{E}\left[\frac{1}{s}\left(X_{i}(s)-x_{i}\right)\left(X_{j}(s)-x_{j}\right) \mid X(0)=x\right] .
$$

The expectations above average the linear and quadratic variation of the process $X(s)$, conditioned on starting at position $x$ at time $s=0$.

If the linear and quadratic time variations on the right hand side of Eqs. (9) and (10) can be computed, a regression problem analogous to Eq. (5) can be formulated and both drift and diffusion can be approximated as an optimal linear combination of basis functions.

\section{Projected dynamics}

In many physical applications, a diffusion process is not observed through its original state space (e.g., atomic coordinates), but through a projected space of lower dimension (e.g., dihedral angles or interatomic distances in macromolecular dynamics). In this case, it is desirable to learn a stochastic dynamical system defined only along the projected variables from the data, often called an effective dynamics, while discarding the other features. We now investigate this issue by following the projection formalism previously proposed in other studies. ${ }^{10,11}$ It is important to note that there are many possible ways of defining an effective dynamics on projected variables (see Ref. 11 for a discussion). In practice, an effective dynamics in the form of an Ito stochastic differential equation (that is, without memory terms) is meaningful if the projected variables capture the slowest dynamical processes and a separation of time scales exists in the system. In the following, we assume this to be the case.

Assume the projection is realized by a map $\xi: \mathbb{R}^{d}$ $\mapsto \mathbb{R}^{m}, m \leq d$ and denote points in the projected space by $z \in \mathbb{R}^{m}$. The level set of a point $z$ is denoted by

$$
\Sigma_{z}=\left\{x \in \mathbb{R}^{d}: \xi(x)=z\right\} .
$$

The projected stationary distribution is obtained by averaging the equilibrium distribution $\mu$ over the level sets $\Sigma_{z}$

$$
v(z)=\int_{\Sigma_{z}} \mu(x) J^{-1 / 2}(x) \mathrm{d} \sigma_{z}(x),
$$

where $J$ is the Jacobian determinant of the transformation $\xi$ and $\sigma_{z}$ denotes the surface measure on the manifold $\Sigma_{z}$. It can be shown ${ }^{11}$ that $v$ defines a probability measure on the low-dimensional space $\mathbb{R}^{m}$. Also, we can define a probability measure $\mu_{z}$ which restricts the equilibrium measure to a level set by

$$
\mathrm{d} \mu_{z}(x)=\frac{1}{v(z)} \mu(x) J^{-1 / 2}(x) \mathrm{d} \sigma_{z}(x),
$$

for $x \in \Sigma_{z}$. Like in Sec. II B 1, an effective dynamics on the lower dimensional space $\mathbb{R}^{m}$ can now be defined by using the Kramers-Moyal formulae

$$
\begin{aligned}
& b_{i}^{\xi}(z)=\lim _{s \rightarrow 0} \mathbb{E}\left[\frac{1}{s}\left(\xi_{i}(X(s))-z_{i}\right) \mid X(0) \sim \mu_{z}\right] \\
& a_{i j}^{\xi}(z)=\frac{\beta}{2} \lim _{s \rightarrow 0} \mathbb{E}\left[\frac{1}{s}\left(\xi_{i}(X(s))-z_{i}\right)\left(\xi_{j}(X(s))-z_{j}\right) \mid X(0) \sim \mu_{z}\right] .
\end{aligned}
$$

The difference between these and Eqs. (9) and (10) is that the dynamics is observed along the projection $\xi$ here and that the initial condition is replaced by starting the process from the distribution $\mu_{z}$ instead of starting deterministically at one point.

\section{Convergence result}

Just as in Sec. II A, we would like to model the components of the (effective) drift and diffusion terms by a linear combination of pre-selected basis functions. Estimation of drift and diffusion from simulation data has been studied for a long time in many different fields of research, like finance, geoscience, climate modeling, and many others. A good overview can be found in Ref. 13. Much attention has been paid to the choice of the discrete time step, see Ref. 14. Spectral estimators have been discussed in Refs. 15 and 16, while estimators for coarse grained dynamics in systems with an explicit multiscale structure can be found in Refs. 17 and 18. In this work, we use a simple linear regression based on the linear and quadratic variations. For infinite data, this regression problem converges to the best-approximation in a function space of the effective drift and diffusion Eqs. (12) and (13). The proof can be found in Appendix B. For ease of notation, we define the $m$-dimensional vector of finite differences as

$$
e^{\xi}(y, x)=\xi(y)-\xi(x) .
$$

Theorem 1. Let $\left\{X\left(t_{1}\right), \ldots, X\left(t_{L+1}\right)\right\}$ be a $d$-dimensional time series from a diffusion process Eq. (7), sampled with an uniform time window $s$. Furthermore, let $\Theta=\left(f_{1}, \ldots, f_{K}\right)$ be a dictionary of basis functions on the projected space $\mathbb{R}^{m}$. Define the database matrix $\mathbb{X}=\Theta\left(X\left(t_{l}\right)\right) \in \mathbb{R}^{L \times K}$ and introduce the set of vectors $\mathbb{Y}_{i}, \mathbb{Z}_{i j} \in \mathbb{R}^{L}$ as

$$
\begin{aligned}
Y_{i, l} & =\frac{1}{s} e_{i}^{\xi}\left(X\left(t_{l+1}\right), X\left(t_{l}\right)\right), \\
Z_{i j, l} & =\frac{\beta}{2 s} e_{i}^{\xi}\left(X\left(t_{l+1}\right), X\left(t_{l}\right)\right) e_{j}^{\xi}\left(X\left(t_{l+1}\right), X\left(t_{l}\right)\right),
\end{aligned}
$$

$\forall i, j=1, \ldots, m$. Then, as $L \rightarrow \infty, s \rightarrow 0$, the solutions $\left\{\tilde{\mathbf{c}}_{i}, \tilde{\mathbf{c}}_{i j}\right\} \in$ $\mathbb{R}^{K}$ of the regression problems

$$
\begin{gathered}
\tilde{\mathbf{c}}_{i}=\underset{\mathbf{c}_{i} \in \mathbb{R}^{K}}{\arg \min }\left\|\mathbb{Y}_{i}-\mathbb{X} \mathbf{c}_{i}\right\|_{2}^{2}, \quad \forall i=1, \ldots, m, \\
\tilde{\mathbf{c}}_{i j}=\underset{\mathbf{c}_{i j} \in \mathbb{R}^{K}}{\arg \min }\left\|\mathbb{Z}_{i j}-\mathbb{X} \mathbf{c}_{i j}\right\|_{2}^{2}, \quad \forall i, j=1, \ldots, m,
\end{gathered}
$$

converge to the coefficient vectors of the best approximation problems

$$
\begin{aligned}
& \tilde{\mathbf{c}}_{i}=\underset{\mathbf{c}_{i} \in \mathbb{R}^{K}}{\arg \min }\left\|b_{i}^{\xi}-\sum_{k=1}^{K} c_{i, k} f_{k}\right\|_{L_{v}^{2}}^{2}, \quad \forall i=1, \ldots, m, \\
& \tilde{\mathbf{c}}_{i j}=\underset{\mathbf{c}_{i j} \in \mathbb{R}^{K}}{\arg \min }\left\|a_{i j}^{\xi}-\sum_{k=1}^{K} c_{i j, k} f_{k}\right\|_{L_{v}^{2}}^{2}, \quad \forall i, j=1, \ldots, m,
\end{aligned}
$$

in the space $L_{v}^{2}$ of square-integrable functions with respect to the measure $v$.

Thus, the optimal linear combinations approximating the drift and diffusion read as

$$
b_{i}^{\xi}=\sum_{k=1}^{K} \tilde{c}_{i, k} f_{k}, \quad a_{i j}^{\xi}=\sum_{k=1}^{K} \tilde{c}_{i j, k} f_{k} .
$$


Please note that the trajectory points $X\left(t_{l}\right), l=1, \ldots$, $L+1$ sample from the $\mu$ equilibrium probability distribution, hence, using Euclidean norms $\|\cdot\|_{2}$ in Eqs. (17) and (18) is appropriate. In fact, a sum over the sampling is equivalent to a $\mu$ weighted sum.

\section{Learning of effective potentials}

In most physical applications, the dynamics of a system is determined by its potential energy, which is a physically intuitive quantity. For example, the overdamped Langevin dynamics in Eq. (8) is defined by the potential energy $U$, which generates the drift via its gradient field. Learning the individual components of the drift separately as in Eq. (17) can pose a challenge in high dimensional systems since there is no guarantee that the learned components are generated by a scalar potential. To circumvent the problem, it is desirable to estimate the potential energy directly instead of its gradient.

For a reversible diffusion process Eq. (7), drift and diffusion define a generalized potential, i.e., there exists a scalar function, which we call free energy $\mathcal{F}: \mathbb{R}^{d} \mapsto \mathbb{R}$ such that ${ }^{19}$

$$
\frac{\partial}{\partial x_{i}} \mathcal{F}(x)=\left[a^{-1}(x)\left(\frac{1}{\beta} \nabla \cdot a_{i}-b\right)\right]_{i} .
$$

Here, we use the notation $\nabla \cdot a_{i}$ to denote the divergence of the $i$ th row of the covariance matrix $a$. Equation (19) also holds for the effective drift and diffusion $b^{\xi}, a^{\xi}$ after applying a projection $\xi$ because the effective dynamics discussed in Sec. II B 2 inherits reversibility from the original dynamics. ${ }^{11}$ Therefore, we discuss the projected case in the following, as estimation of the full dynamics is a special case of this problem.

Since the gradient of $\mathcal{F}$ in Eq. (19) now depends on two unknowns, we need to estimate one of them first before we can solve for the free energy gradient. Suppose we have used the regression of Eq. (18) to obtain an expression for each component of the diffusion matrix $a^{\xi}(x)$ as

$$
a_{i j}^{\xi}(x)=\sum_{k=1}^{K} c_{i j, k} f_{k} .
$$

This model allows us to evaluate each component of the diffusion and its derivatives at every simulation point. At this point, we assume that the model Eq. (20) still defines a positive definite matrix field, which is the case for the applications we discuss later (Sec. V). Positive definiteness of the diffusion could also be enforced in the optimization problem, but that would lead to a more involved problem than the standard linear regression we consider here.

Next, we can use Eq. (19) and the convergence of linear variations to the effective drift to set up a regression problem for the free energy gradient as a linear combination of the vector fields $\nabla f_{k}$, i.e.,

$$
\nabla \mathcal{F}=\sum_{k=1}^{K} v_{k} \nabla f_{k}
$$

The regression problem becomes (see Appendix C)

$$
v=\underset{w \in \mathbb{R}^{K}}{\arg \min }\|\mathbb{Y}-\mathbb{D} w\|_{2}^{2},
$$

where $\mathbb{Y}$ and $\mathbb{D}$ are now given by

$$
\begin{aligned}
\mathbb{Y}_{i l} & =\left[\left(a^{\xi}\right)^{-1}\left(X\left(t_{l}\right)\right)\left(\frac{1}{\beta} \nabla \cdot a_{i}^{\xi}\left(X\left(t_{l}\right)\right)-\frac{1}{s} e^{\xi}\left(X\left(t_{l+1}\right), X\left(t_{l}\right)\right)\right)\right]_{i}, \\
\mathbb{D}_{i l, k} & =\frac{\partial f_{k}}{\partial z_{i}}\left(X\left(t_{l}\right)\right) .
\end{aligned}
$$

\section{METHODS}

Our goal is to find a sparse representation to the drift and diffusion term, which requires computing a sparse solution to the regression problem Eqs. (17) and (18). Standard regression can be biased toward sparse solutions by introducing a $L^{0}$ constraint (also known as subset selection) into the standard optimization process

$$
\tilde{\mathbf{c}}=\underset{\mathbf{c}}{\arg \min }\left(\|\mathbb{Y}-\mathbb{X} \mathbf{c}\|_{2}^{2}+\rho\|\mathbf{c}\|_{0}\right),
$$

where the 0-norm $\|\mathbf{c}\|_{0}$ denotes the number of non-zero components in $\mathbf{c}$.

Unfortunately, the minimization problem Eq. (22) is nonconvex, which makes finding a solution a NP-hard task. One popular way around this problem is to relax the problem to a $L^{1}$-norm constraint, and a sparse solution can then be computed by using one of the many algorithms available, such as Lasso, ${ }^{5,20}$ matching pursuit ${ }^{21}$ and its orthogonal variant $\mathrm{OMP},{ }^{22}$ or elastic net, ${ }^{23}$ just to name a few. Independently of the specific protocol, the solution will have some coefficients equal to zero.

In principle, any sparsity value can be enforced in the solution, by tuning the Lagrange multiplier $\rho$ in Eq. (22). However, only a subset of these values provides a representation of the data set that is both accurate and compact. For instance, we expect an excessively sparse solution c to severely under-fit and a barely sparse solution to over-fit the data. ${ }^{24}$ An under- (over-)fitted model contains less (more) parameters than can be justified by that data, and both regimes should be avoided. For this reason, any algorithm enforcing sparsity needs to be complemented by a criterion that allows us to assess whether a solution is still statistically meaningful and that signals whether the over-fitting or under-fitting regimes are entered, in order to automatically select the sparsity level. We propose to use the statistical procedure of Cross Validation ${ }^{25}$ to select solutions with optimal sparsity.

We show in the following that when using Cross Validation sparsity can be automatically enforced with iterative algorithmic formulations such as the Stepwise Sparse Regressor (SSR). Despite its intrinsic simplicity and intuitive interpretation, such an algorithm appears to be robust and effective, as it is discussed below.

\section{A. Sparsity enforcement}

The approach we employ to solve the sparse regression Eq. (22) for stochastic systems is inspired by the iterative thresholding algorithm proposed by Brunton et al. in their deterministic SINDy study, ${ }^{4}$ which works as follows. First, a standard unconstrained linear regression is solved to compute a non-sparse solution $\mathbf{c}$. Then, coefficients with a magnitude smaller than a pre-defined threshold value $\lambda$ are set to zero and 
regression is performed on the remaining coefficients, and the procedure is iterated till no coefficients are found smaller than $\lambda$. The threshold parameter $\lambda$ is a sparsification knob which needs to be tuned appropriately.

We modify the thresholding approach to enforce sparsity iteratively by removing only one coefficient in every iteration and use Cross Validation to select the number of iterations, as it is discussed in Sec. III B. This modification removes the need of adjusting an external parameter like $\lambda$. The pipeline works as follows:

- A standard least square regression,

$$
\tilde{\mathbf{c}}=\underset{\mathbf{c} \in \mathbb{R}^{K}}{\arg \min }\|\mathbb{Y}-\mathbb{X} \mathbf{c}\|_{2}^{2},
$$

is solved to determine a preliminary (non-sparse) solution $\tilde{\mathbf{c}}$.

- One coefficient is set to zero. Different criteria can be used in principle to select which coefficient to remove at each iteration. Here, the one coefficient with the smallest absolute value is set equal to zero, i.e.,

$$
\tilde{c}_{i}=0: i=\min _{k}\left|\tilde{c}_{k}\right| .
$$

This particular choice resembles the thresholding protocol proposed in Ref. 4, with the additional advantage that the solution sparsity equals the number of iterations on which the algorithm is being run, which makes solution sparsity programmable exactly.

- Standard regression is performed again on the remaining degrees of freedom

$$
\mathbb{Y}=\mathbb{X}[:, \hat{i}] \tilde{\mathbf{c}}[\hat{i}],
$$

where the colon notation $\mathbb{X}[:, k]$ identifies the $k$ th column of the matrix and $\hat{\imath}$ indicates the set of all dictionary indexes but $i$, which has been removed.

- The procedure is iteratively repeated until Cross Validation indicates that the optimal sparsity level (i.e., the number of iterations) $\tilde{s}$ in the solution $\tilde{\mathbf{c}}$ is reached.

We call this algorithm the Stepwise Sparse Regressor (SSR) and introduce the shorthand notation

$$
\operatorname{SSR}(\mathbb{X}, \mathbb{Y})_{q}
$$

to indicate the solution $\mathbf{c}$ obtained upon running the algorithm on $q$ iterations. Such a solution is $q$-sparse, e.g., has $q$ zero coefficients and $n=K-q$ non-zero coefficients. In the following, we are going to refer to the parameter $n$ as the solution size, while discussing the results.

Once Cross Validation is used to identify the number of iterations corresponding to the optimally sparse solution, the algorithm is parameter free and does not require any preliminary training phase before use.

A complete SSR run may require a large number of regressions if the basis is large and the final solution is very sparse. However, producing the simulation data remains the main computational burden. Alternative build-up approaches such as Orthogonal Matching Pursuit ${ }^{22}$ have been investigated, but appeared to be less robust, in that it would get stuck in suboptimal minima more readily than the SSR.

\section{B. Cross validation}

The specific number of iterations on which the Stepwise Sparse Regression needs to be run to find the optimal solution is determined by a Cross Validation (CV) calculation, ${ }^{26}$ a statistical validation technique that has risen to great popularity in the interdisciplinary fields of model and hyperparameter selections (see Ref. 24 for an introductory self contained discussion). The underlying idea is straightforward and summarized below.

Let us assume, we have a family of parametric models $\left(\mathcal{M}\left(\lambda_{1}\right), \ldots, \mathcal{M}\left(\lambda_{r}\right)\right)$ depending on a hyperparameter $\lambda$ which takes values $\lambda_{1}, \ldots, \lambda_{r}$ and we would like to select the one model that fits best a given data set $\mathcal{D}$. In the original $\mathrm{CV}$ formulation, the full data set is split into two disjoint subsets and each model in the family is alternatively trained on one of them first and then tested on the other. The cross validation score is the average deviation $\delta$ of the predictions of the trained model from the actual test set, and it measures how accuracy and predictivity are balanced in that model. The set of parameters yielding low values of $\delta$ are selected and identify "optimal" models in the family.

Here, we use CV to select the size $n$ of the optimal solution to the linear regression problem Eq. (22), which plays the role of the hyperparameter $\lambda$ from the last paragraph. The family of models to validate is now a set of SSRs with different solution size $n=K-q$ (or, equivalently, different sparsity $q$ ), i.e.,

$$
\left\{\operatorname{SSR}_{q}\right\}_{q=1, \ldots, K},
$$

where the notation introduced in Sec. III A is used. CV is run on each model to generate a family of cross validation scores $\delta\left[S S R_{q}\right]$.

We use a $k$-fold cross validation formulation, where the full dataset is split into $k$ batches, each of them playing alternatively the role of the test set in a $k$ step procedure. Let us start by partitioning the dataset $\mathcal{D}$ containing $N$ data points $p$ into $k$ disjoint equivalent subsets $A_{i}$, which are selected randomly, i.e., $\bigcup_{i} A_{i}=\mathcal{D}, A_{i} \cap A_{j}=\emptyset$. Moreover, let us introduce the shorthand

$$
\mathbb{X}_{A_{i}}=\mathbb{X}\left[p_{A_{i}},:\right], \quad p_{A_{i}}=\bigcup_{p \in A_{i}} p,
$$

where the notation $\mathbb{X}[k,:]$ indicates the $k$ th row of the matrix. Then, the cross validation score for each model $S S R_{q}$ is defined as an average

$$
\begin{gathered}
\delta^{2}\left[S S R_{q}\right]=\frac{1}{k} \sum_{i=1}^{k}\left\|\mathbb{Y}_{A_{i}}-\mathbb{X}_{A_{i}} \cdot \operatorname{SSR}\left(\mathbb{X}_{B_{i}}, \mathbb{Y}_{B_{i}}\right)_{q}\right\|_{2}^{2}, \\
B_{p}=\bigcup_{i \neq p} A_{i},
\end{gathered}
$$

where $\operatorname{SSR}\left(\mathbb{X}_{B_{i}}, \mathbb{Y}_{B_{i}}\right)_{q}$ indicates the $q$-sparse linear combination coefficients generated by running $S S R$ on the training set $B_{i}$ [see notation Eq. (23)], which are then used to make a prediction $\mathbb{X}_{A_{i}} \cdot \operatorname{SSR}\left(\mathbb{X}_{B_{i}}, \mathbb{Y}_{B_{i}}\right)_{q}$. The set of $\delta\left[S S R_{q}\right]$ is then monitored as a function of the solution size $n=K-q$, which usually results in a behavior close to that represented in Fig. 1. We expect an intermediate regime of low cross validation score (accurate) solutions [region (2)] with variable sparsity: all such solutions 


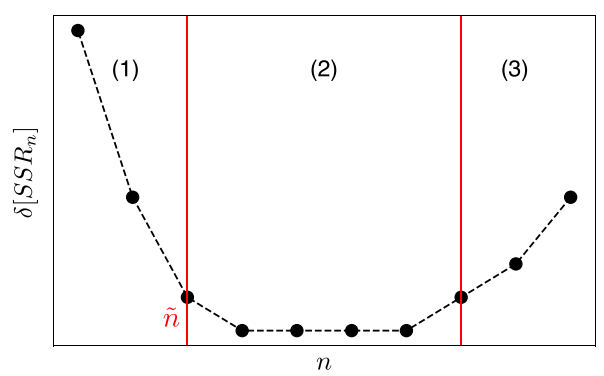

FIG. 1. Cartoon representation of the expected behavior of the $k$ fold cross validation score $\delta\left[S S R_{n}\right]$ as a function of the solution size $n$ in the linear combination solution.

are equally good at balancing sparsity and accuracy. In addition, this regime is bounded from the right and left by and under- and over-fitting regimes [regions (1) and (3)], respectively. Solutions belonging to both regimes are characterized by larger values of the cross validation scores, which indicate that accuracy is decreasing. Intuitively, the one solution separating regime (2) from regime (1) is what we call optimally sparse $\tilde{n}$ since

$$
\frac{\delta[\tilde{n}-1]}{\delta[\tilde{n}]} \gg 1, \quad \frac{\delta[\tilde{n}]}{\delta[\tilde{n}+1]} \approx 1 .
$$

The $\tilde{n} \rightarrow \tilde{n}+1$ gap in the $\delta$ values is a clear signal that increasing sparsity by one additional unit compromises the model predictive power.

In the following, the optimally sparse solution $\tilde{n}$ is chosen by identifying such a transition point in the cross validation score curves. Please note that the over-fitting regime (3) may be absent if the database of chosen functions in the dictionary is not very redundant. On the contrary, under-fitting can always be accomplished. An even scarcer and poorer dictionary would immediately result in both regimes (2) and (3) missing from plot Fig. 1, the model being heavily under-fitted.

All cross validation calculations reported below were performed using Python routines available in sklearn. ${ }^{27}$

\section{HOMOGENEOUS DIFFUSION IN A DOUBLE WELL POTENTIAL}

We illustrate our sparse regression protocol by applying it to overdamped homogeneous diffusion data in a one dimensional double well polynomial potential Fig. 2

$U(x)=\frac{1}{2} x^{4}-4 x^{3}+9 x^{2}-3 x, \quad \frac{d U}{d x}=2 x^{3}-12 x^{2}+18 x-3$,

to recover the drift of the process from the data. Five independent trajectories were generated by integrating Eq. (8) using the gradient in Eq. (26). We computed the time increments, Eq. (15), and averaged them over discrete bins as explained in Appendix A 1. We illustrate the performance of the sparse regression on two different dictionaries $\Theta$ and $\Theta^{\prime}$. Each dictionary consists of $K=20$ basis functions of the form [1, $x, x^{2}$, $\left.x^{3}, \ldots\right]$. Details about the dataset and the specific composition of the two dictionaries are provided in Appendix A, Eqs. (A2) and (A3). The first four entries in both databases correspond to the functions composing the drift term used to generate the data $\left[1, x, x^{2}, x^{3}\right]$. We refer to these four functions as analytic basis functions in the following.

Figure 3(a) shows the cross validation score $\delta_{\Theta}$ from Eq. (25) for the first dictionary, $\Theta$, as a function of the solution size $n$. As the number of surviving coefficients $n$ decreases (from right to left), the cross validation score stays constant; however, going from a four term $n=4$ to a three term $n=3$ solution causes the cross validation score to increase by several orders of magnitude, which suggests that sparsity is now too extreme and compromises the model predictivity. The signal is clear, as indicated by the plot of ratios $\delta[n-1] / \delta[n]$ as a function of $n$ [inset in Fig. 3(a)]. The position of the gap in the CV score curve suggests that $n=4$ is the optimal solution sparsity, which is associated with a $\tilde{\delta}_{\Theta}=1.49 \cdot 10^{-4} \mathrm{CV}$ score.

Figure 3(b) shows the sparsity progress matrix, which monitors the linear combination status as a function of sparsity. The $(i, j)$ entry in the matrix refers to the function $f_{i}$ in the dictionary, when the solution only contains $n=j$ terms (i.e., after $20-n$ iterations). The color code is as follows: gray pixels indicate that the coefficient $c_{i}$ is still alive, whereas white pixels are used for coefficients that have been removed. A horizontal black line indicates the optimal solution size $\tilde{n}$, as from the cross validation score plot, and the corresponding pixels are also colored in black. A light blue color is used to mark the analytic basis functions. Please note that no coefficient is resuscitated during the SSR iterations after it is removed from the dictionary in a previous iteration.
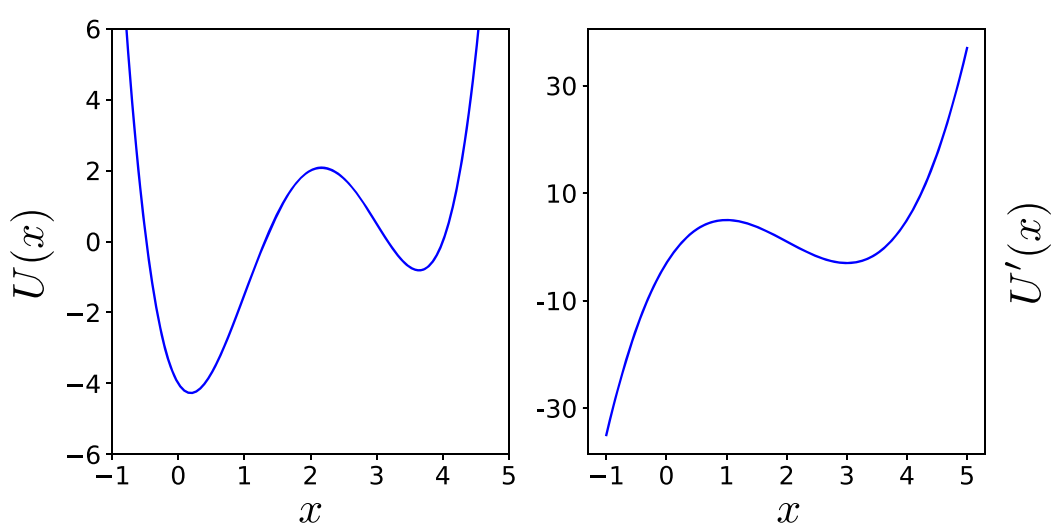

FIG. 2. Potential energy profile $U(x)$ (left panel) and its gradient $U^{\prime}(x)$, which equals the opposite of the force (right panel). 
(a)

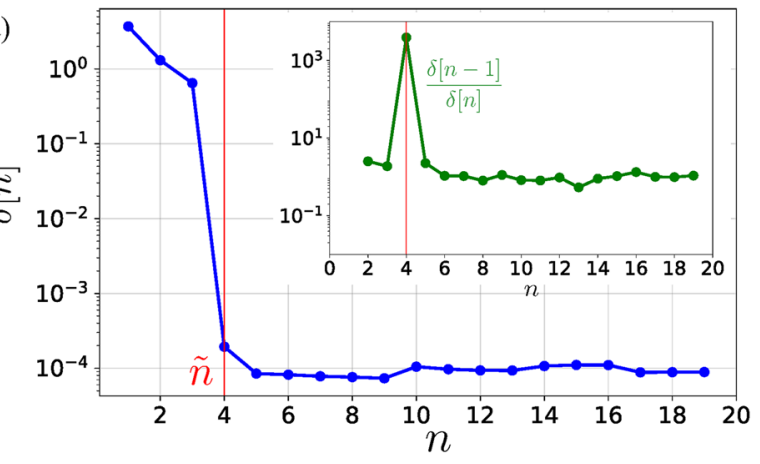

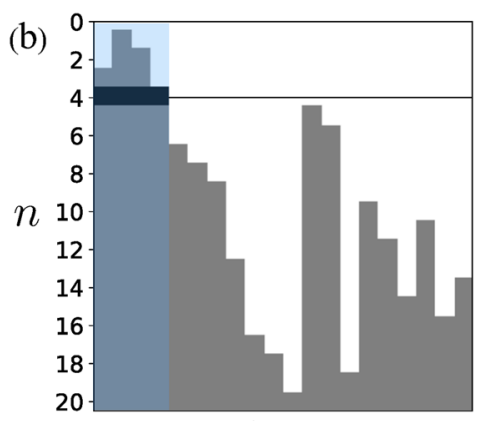

basis function idx
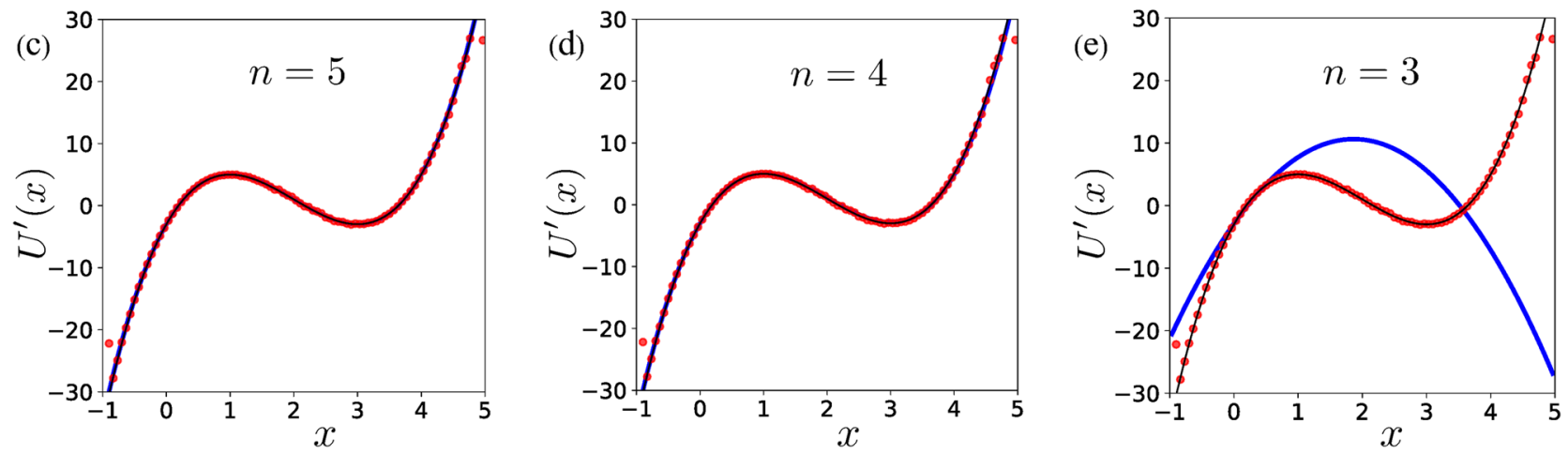

FIG. 3. Results from applying the $S S R$ algorithm to a trajectory generated by diffusion in the potential of Eq. (26), using a function dictionary $\Theta$. [Panel (a)] Cross validation score is plotted as a function of solution size $n$. The inset shows the ratio $\delta[n-1] / \delta[n]$ as a function of $n$. Vertical red lines indicate the number of non-zero coefficients $\tilde{n}=4$ in the optimal solution. [Panel (b)] Sparsity progress matrix: any $(i, j)$ (gray) white entry indicates that coefficient $c_{i}$ is (non)-zero after $K-j(K=20)$ iterations. A horizontal black line indicates $\tilde{n}$ and the corresponding coefficients are colored in black. The four dictionary basis functions $\left[1, x, x^{2}, x^{3}\right]$ have survived and are highlighted in a blue color. [Panels (c)-(e)] Comparison between the exact gradient $U^{\prime}(x)$ (black solid line) and solutions $\tilde{U}^{\prime}(x)=\sum_{k} \tilde{c}_{k} f_{k}(x)$ (blue solid lines) with decreasing solution size $n$ values (or increasing sparsity, from left to right). Red markers represent the binned time increments as from Eq. (9).

The optimal solution only contains the analytic basis functions and reads as

$\tilde{U}_{\Theta}^{\prime}(x)=\tilde{\mathbf{c}} \cdot \Theta(x)=-2.98+17.84 x-11.82 x^{2}+1.96 x^{3}$.

This expansion is an accurate approximation to Eq. (26), as it can be seen by comparing coefficients and from panel (d) in Fig. 3.

It is instructive to compare the optimal solution Eq. (27) to a slightly less sparse $n=5$ and to a sparser $n=3$ solution. The explicit expansions for these cases are

$$
\begin{aligned}
& U_{n=5}^{\prime}(x)=-2.98+17.43 x-11.75 x^{2}+1.98 x^{3}+0.4 \sin x, \\
& U_{n=3}^{\prime}(x)=-2.88+14.5 x-3.89 x^{2},
\end{aligned}
$$

and are plotted together with the gradient Eq. (26) in Figs. 3(c) and 3(e). The $n=5$ solution contains the analytic functions and an extra small oscillatory term and accurately approximates the gradient, $\delta=7.8 \cdot 10^{-5}$. By contrast, the sparser solution $n=3$ is deprived of one key dictionary ingredient and does not perform well, as shown in Fig. 3(e) and the much larger cross validation score $\delta=9 \cdot 10^{-1}$.

Let us now discuss the solution to the problem Eq. (9) when a different dictionary $\Theta^{\prime}$ is used. Results are summarized in Fig. 4, using the same format as in Fig. 3.

The cross validation score plot in Fig. 4(a) shows a similar trend as in Fig. 3(a), but a clear gap in the $\delta$ values is now missing and suggests that an optimal solution is somewhere in the range between $n=5$ and $n=10$. The transition point $\tilde{n}_{k}^{\prime}=7$ is selected as the value of $n$ maximizing the $\mathrm{CV}$ score ratio $\delta[n-1] / \delta[n]$, as shown in the inset. It is worth noting that the maximum amplitude of the ratio is here two orders of magnitude smaller than in Fig. 3(a). The progress matrix in Fig. 3(b) shows that the three analytic terms $\left[x, x^{2}, x^{3}\right]$ are present in the optimal solution, but $f_{0}=1$ is not (first column), and there are additional contributions. The actual expansion $\tilde{U}^{\prime}(x)=\tilde{\mathbf{c}}^{\prime} \Theta^{\prime}(x)$ reads as

$$
\begin{aligned}
\tilde{U}^{\prime}(x)= & 17.85 x-12.68 x^{2}+2.11 x^{3}+9.61 \exp \left(-50(x-3)^{2}\right) \\
& -2.97 \exp \left(-50(x-4)^{2}\right)-18.77 \exp \left(-0.6(x-4)^{2}\right) \\
& +12.97\left[\tanh ^{2}(x-4)+1\right] .
\end{aligned}
$$

Performing a CV score based SSR on the two different dictionaries produces two solutions with different levels of sparsity and cross validation scores. Both of them succeed at capturing the double well feature Figs. 3(c) and 4(c) of the potential Eq. (26). As a matter of fact, a Taylor expansion of the $\Theta^{\prime}$-solution Eq. (30) returns a polynomial series which is in consistent with Eq. (27).

However, the solution associated with dictionary $\Theta^{\prime}$ is less parsimonious than what was found for the dictionary $\Theta$ considered above, and it is associated with a larger cross validation score

$$
\tilde{\delta}_{\Theta}=1.49 \cdot 10^{-4}<\tilde{\delta}_{\Theta^{\prime}}=5.6 \cdot 10^{-3} .
$$



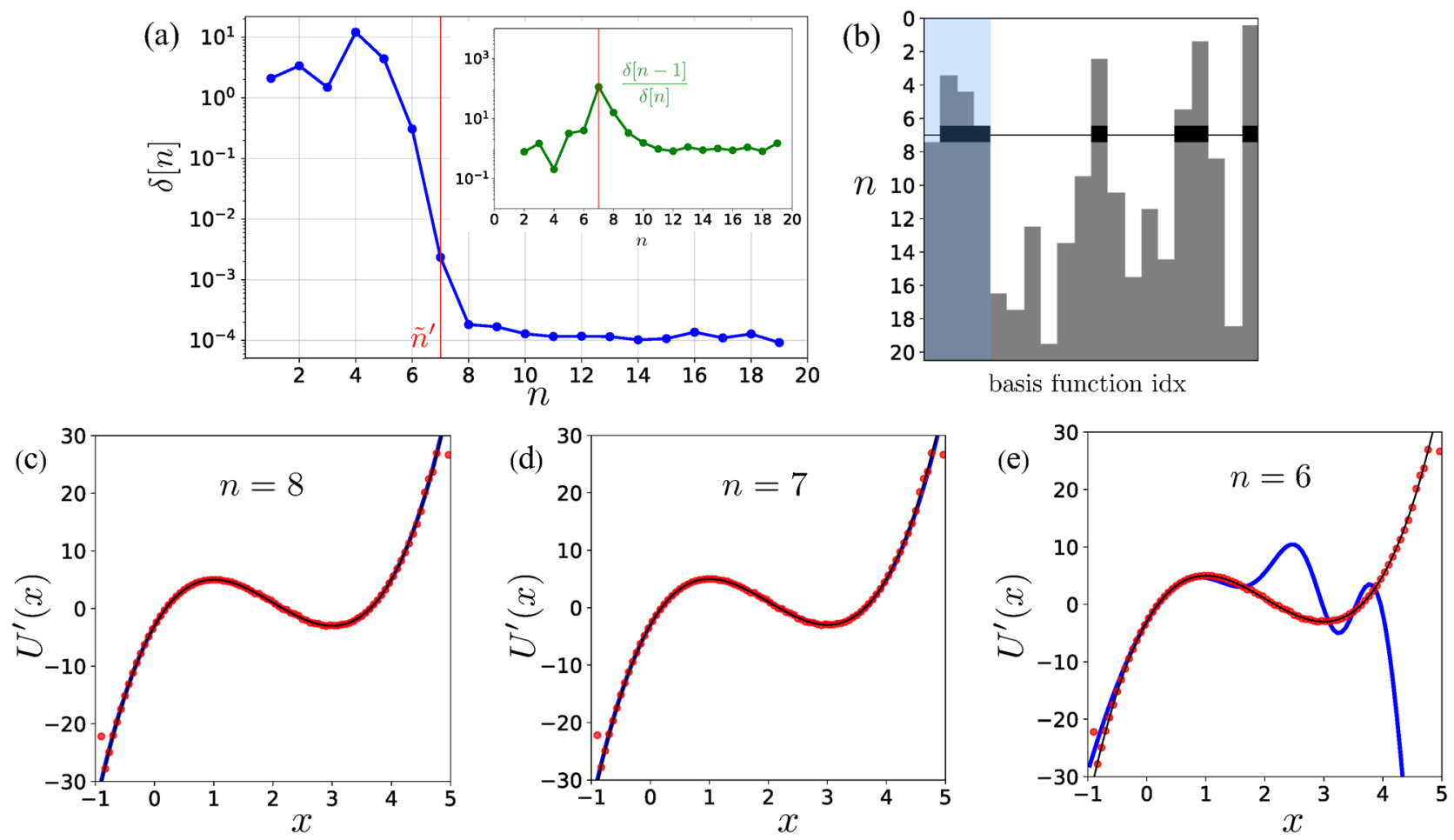

FIG. 4. Sparse regression results for a dictionary $\Theta^{\prime} \neq \Theta$, using the same notation and color code as in Fig. 3. [Panel (a)] Cross validation score as a function of solution size $n$. The inset shows the ratio $\delta[n-1] / \delta[n]$ as a function of $n$. Vertical red lines indicate the number of non-zero coefficients $\tilde{n}^{\prime}=7$ in the optimal solution. [Panel (b)] Sparsity progress matrix. [Panels (c)-(e)] Comparison between the exact gradient $U^{\prime}(x)$ (black solid line) and solutions $\tilde{U}^{\prime}(x)=\sum_{k} \tilde{c}_{k}^{\prime} \Theta^{\prime}(x)$ (blue solid lines) with decreasing solution size $n$ (or increasing sparsity, from left to right). Red markers represent the binned time increments as from Eq. (9). Even though $\tilde{c} \neq \tilde{c}^{\prime}$, the gradient is still accurately approximated.

For this reason, the solution for dictionary $\Theta$ should be favored over $\Theta^{\prime}$. We show in Sec. IV A that even if different dictionaries return different optimal solutions, the comparison of results for different dictionaries leads to the identification of the maximally sparse solution.

\section{A. Greedy search}

The results discussed above indicate that the performance of the SSR algorithm is affected by the composition of the dictionary used. The resulting optimal solutions are quantitatively different but qualitatively very similar to one another. This section is devoted to investigating this issue systematically.

The comparison of Figs. 3 and 4 shows that the cross validation signature when the SSR identifies that the correct (maximally sparse) solution is much stronger than for the case of a less sparse solution.

This consideration suggests that, instead of proceeding by iteratively removing functions from the dictionary, cross validation could be used to extensively test all possible combinations of basis functions and compare the results to determine the maximally sparse solution. That is, given a large reference dictionary, all possible combinations of functions (with a given sparsity) could be considered and the corresponding CV-score estimated and compared.

We illustrate this idea by defining a large reference dictionary $\Omega$ of $M=100$ basis functions. The two previously used dictionaries are included in this large one $\Theta, \Theta^{\prime} \subset \Omega$. The standard (non-sparse) linear regression problem,

$$
\mathbf{c}_{\theta}=\underset{\mathbf{c}}{\arg \min }\left\|\mathbb{Y}-\mathbb{X}_{\theta} \mathbf{c}\right\|_{2}^{2},
$$

can be solved for each sub-dictionary $\theta \subset \Omega$ with $n<M$ functions, and the associated cross validation score $\delta_{\theta}$ can be computed. If the $\delta_{\theta}$ values for all possible sub-dictionaries $\theta \subset \Omega$ and different $n$ values are tabulated, the optimal solution can be identified by comparing the change in cross validation as a function of the dictionary size. Such a procedure evaluates and compares different levels of sparsity in a greedy fashion and can in principle replace the need of an iterative sparse regression algorithm.

For a very large reference database of $M$ functions, such a brute force approach becomes computationally very demanding as the number of different sub-dictionaries with $n$ functions is given by the binomial coefficient $C_{M, n}=\left(\begin{array}{c}M \\ n\end{array}\right)$, which becomes untreatable if $n \gg 1$. In order to demonstrate the approach, we randomly sample $m_{n}$ different $n$-function dictionaries $\theta$ from $\Omega$, for increasing values of $n$, and run $\mathrm{CV}$ validation on each of them (details on specific values of $m_{n}$ are given in Appendix A).

The main results are shown in Fig. 5, where the cross validation scores $\delta_{\theta}$ (averaged over several realizations, as detailed in the Appendix) are plotted as a function of the dictionary size $n$.

Each dictionary realization is described by an orange point $\left(n, \delta_{\theta}\right)$, unless the analytic basis functions $[1, x$, $\left.x^{2}, x^{3}\right] \subset \theta_{n}$, in which case the point is blue. The minimum cross validation score was selected (marked in black in Fig. 5) across all points for each dictionary size $n$, 


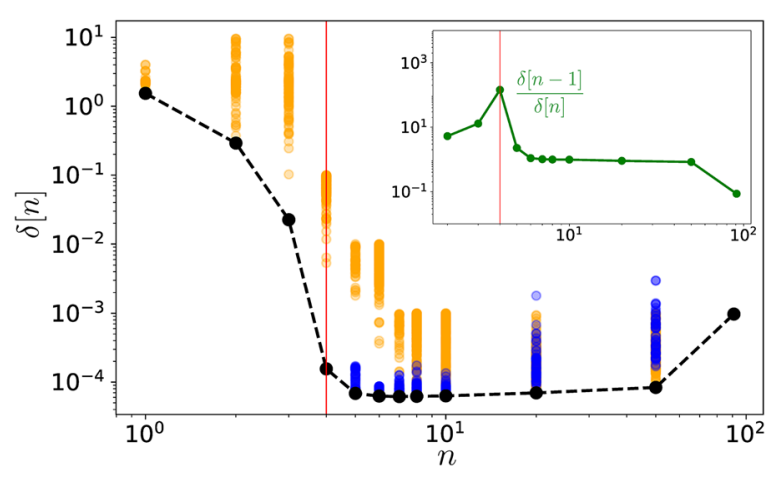

FIG. 5. Greedy search in the solution space to the regression problem, Eq. (22), applied to the two-well potential, Eq. (26). For a given size $n$, the cross validation score for a set of 100 different dictionaries $\delta_{\theta, n}$ (subsets of the large reference dictionary $\Omega$ ) are plotted. Each marker represents a dictionary instance, and is colored in blue if the dictionary contains the analytic basis functions $\left[1, x, x^{2}, x^{3}\right] \subseteq \theta$, or in orange otherwise. The minimal (optimal) cross validation score min $\left(\delta_{k}\right)$ over all realizations with a given size $n$ is indicated with a black dashed line. A vertical red line indicates the solution with optimal sparsity, as determined by the peak in the function $\delta[n-1] / \delta[n]$, as shown in the inset and represents the optimally sparse solution $\tilde{\theta}=\left[1, x, x^{2}, x^{3}\right]$.

and the resulting curve is plotted using a dashed black line.

Figure 5 shows that the optimal sparse solution $\tilde{\theta}=\left[1, x, x^{2}, x^{3}\right]$, which is associated with $\delta=1.49 \cdot 10^{-4}$ (as also obtained for the $\Theta$-solution in Sec. IV), clearly represents the transition point in the cross validation curve. Any other $n=4$ dictionary has a larger cross validation score, as signaled by the gap between the blue point and all other orange realizations at $n=4$. Moreover, any sparser $(n=3)$ dictionary has an associated cross validation score larger by several orders of magnitude. The increase in CV score is a footprint that a dictionary is missing (at least) a key component (as in the cross validation score plots in Figs. 3 and 4). As already observed before, less sparse solutions such as $5<n<20$ may reproduce the gradient of the potential equally good or slightly better and they all have comparable cross validation scores. When $n \gg 1$ (as the size of the dictionary increases), the cross validation scores start increasing, indicating the over-fitting regime.

The $\Theta^{\prime}$ solution that was found in Fig. 4 is represented by a $n=7$ orange point in Fig. 5, together with many others that share the same sparsity.

This greedy analysis shows that cross validation identifies the analytic basis functions $\tilde{\theta}=\left[1, x, x^{2}, x^{3}\right]$ as the optimal sparse solution to the problem Eq. (17); sparser solutions are less accurate (larger $\delta$ ), and comparably accurate solutions are less parsimonious (reduced sparsity), as indicated also by the plot of $\delta[n-1] / \delta[n]$ as a function of solution size $n$ (the inset in Fig. 5).

This analysis calls attention to the shortcoming of the deterministic SSR algorithm to search for the optimal solution. As seen in Sec. IV, the performance of SSR depends on the choice of the database. The deterministic nature of SSR is not always efficient in searching the solution space of the non-convex problem and SSR can be trapped in local minima that provide a sub-optimal solution.
The performance of the SSR algorithm cannot be a priori estimated by considering indicators of the ill-conditioning of the dictionary. For the example of the two dictionaries $\Theta$ and $\Theta^{\prime}$ used in Sec. IV, the condition number $\kappa$ of the database does not reflect their performance, as

$$
\kappa\left(\mathbb{X}_{\Theta}\right) \approx 10^{7}, \quad \kappa\left(\mathbb{X}_{\Theta^{\prime}}\right) \approx 10^{3} .
$$

At the level presented here, the proposed CV-based SSR is effective at relaxing to a sparse (even if not always the sparsest) solution, which is dictionary dependent but which still efficiently captures the main features of the gradient.

\section{B. Effects of sampling noise on algorithmic performance}

In this section, we investigate to what extent the convergence of the sparse regression algorithm is influenced by the presence of noise in the stochastic system, by using the double well potential Eq. (26) as a reference system. One of the main assumptions underlying the proof of Theorem 1 is that the data points are Boltzmann distributed. However, this condition is met only approximately on a finite size trajectory.

In order to investigate how deviations from the Boltzmann distribution affect the performance of the algorithm, we bin the trajectory along the $\mathrm{x}$-axis in $Q$ bins and introduce the bin-dependent relative error

$$
\epsilon_{i}=\left|\frac{Y_{i}-\bar{U}^{\prime}\left(x_{i}\right)}{\bar{U}^{\prime}\left(x_{i}\right)}\right|,
$$

where $Y_{i}$ is the average of the time increments, Eq. (15), associated with bin $i$, and $\bar{U}^{\prime}\left(x_{i}\right)$ indicates the average value of the gradient across all trajectory points $x_{i}$ in bin $i$. The median of the error distribution $\left\{\epsilon_{i}\right\}_{i=1, \ldots, Q}$ over the trajectory sample used in Sec. IV is approximately $10^{-2}$ and the largest deviation is found for the bin located on the top of the energy barrier (see Fig. 2). The error could be decreased by combining a lower simulation temperature with longer production times. Here we mimic these effects by generating new samples $\hat{Y}_{i}$ in every bin $i$ with increasingly smaller deviation from the ideal sample, using a scaling factor $f<1$

$$
\hat{Y}_{i}^{f}=\mathcal{N}\left(\bar{U}^{\prime}\left(x_{i}\right), \zeta\right), \quad \zeta=f \cdot \operatorname{median}(\epsilon) .
$$

The noise distribution $\mathcal{N}$ was chosen to be Gaussian to approximate the effect of thermal noise. In practice, the same effect could be obtained by running umbrella sampling simulations in every bin. The sparse regression was then run on this new, less noisy data set, for 100 different random 50 basis function dictionaries $\Theta$ (all including $\left[1, x, x^{2}, x^{3}\right]$ entries) and different values of the scaling parameter $f$. The performance of the SSR

TABLE I. Percentage of dictionaries for which SSR converges to the optimal solution $\left[1, x, x^{2}, x^{3}\right]$, as a function of the noise scaling factor $f$, Eq. (31). $f=-\infty$ is a shorthand indicating no sampling noise.

\begin{tabular}{llllll}
\hline \hline $\log _{10} f$ & 0 & -3 & -6 & -9 & $-\infty$ \\
\hline$\%$ & 0 & 0 & 46 & 81 & 94 \\
\hline \hline
\end{tabular}


algorithm as a function of the sampling error is reported in Table I.

While for large sampling errors the SSR algorithm converges to the optimal solution $\left[1, x, x^{2}, x^{3}\right]$ for none of the 100 randomly selected dictionaries, the percentage of dictionaries where the optimal solution is found by the SSR increases as the sampling noise is reduced. Surprisingly, a handful of dictionaries still relax to a sub-optimal minimum even in the absence of noise.

This result indicates that for stochastic systems the sampling quality plays an important role in determining whether the SSR algorithm gets trapped in a local minimum in the solution space. Additionally, the effect of the noise reduction is dictionary-specific, as some dictionaries do not reach the global minimum till extremely low level of noise.

In practice, relevant (usually high-dimensional) systems will present large sampling errors and a more robust algorithm for the search in the solution space is needed.

\section{V. "LEARNING" A PROJECTED DYNAMICS}

We apply the analysis protocol discussed in Secs. II-III to "learn" sparse representations for the drift and diffusion coefficients of a stochastic dynamics along an effective coordinate, ${ }^{10}$ starting from a pre-determined library of basis functions. As a benchmark, we use a system where the solution can be computed analytically, that is, the two dimensional lemon slice potential introduced by Bittracher et al. ${ }^{28}$ which is specified by the polar representation $(\theta, r)$,

$$
U(r, \phi)=\cos (\kappa \phi)+10(r-1)^{2}+\frac{1}{r}
$$

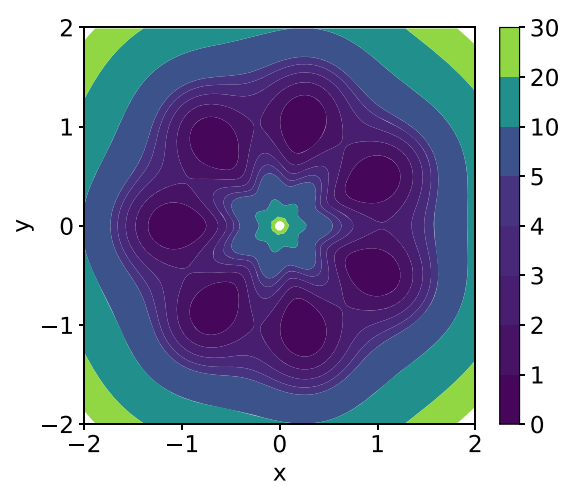

FIG. 6. A contour plot $U=U(x, y)$ of the lemon slice potential Eq. (33) is shown. Seven angular mimima $\kappa=7$ can be clearly distinguished. Details are given in the text.

where $\kappa=7$ indicates the number of minima in the energy landscape, as shown in Fig. 6.

It was previously shown ${ }^{28}$ that the polar angle $\phi \in[-\pi$, $\pi$ ] correlates with the first seven eigenvectors of the backward Fokker Planck operator associated with this potential, which describe the basin hopping motions. The polar angle $\phi$ is then a good candidate for an effective coordinate. Projecting the overdamped diffusion into this coordinate results in the projected dynamics

$$
\begin{gathered}
d \phi_{t}=b(\phi) d t+\sqrt{2 \beta^{-1} a(\phi)} d \eta(t), \\
b(\phi)=\frac{C_{r}}{Z_{r}} \kappa \sin \kappa \phi, \quad a(\phi)=\frac{C_{r}}{Z_{r}},
\end{gathered}
$$

with the constants (a) $10-1$

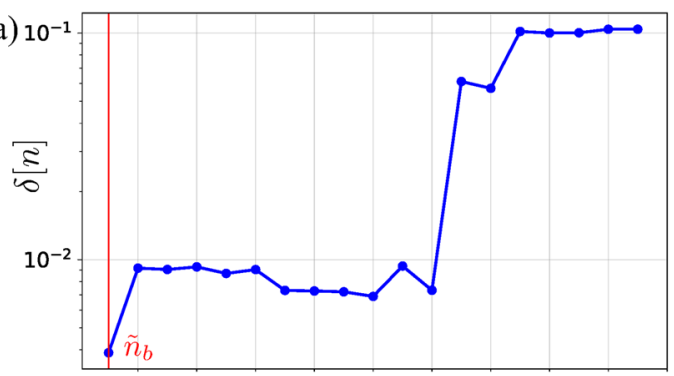

(d)

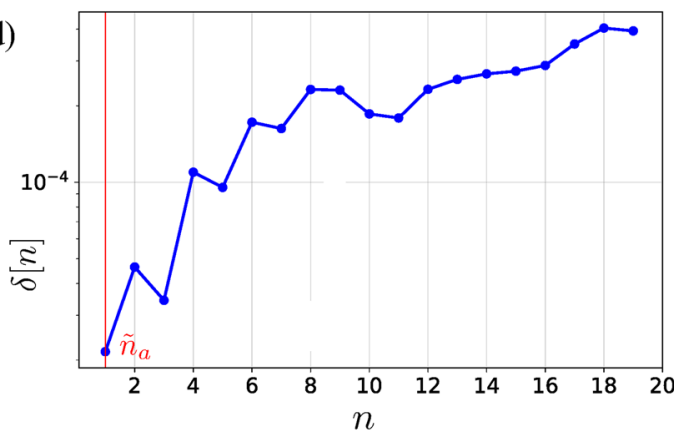

(b)
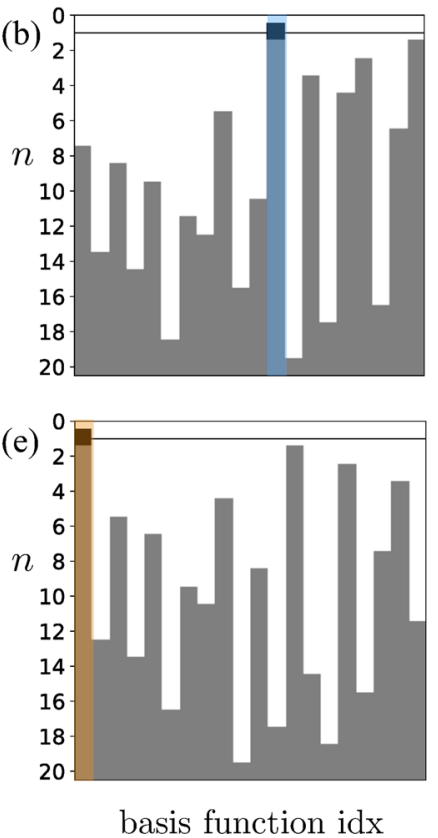
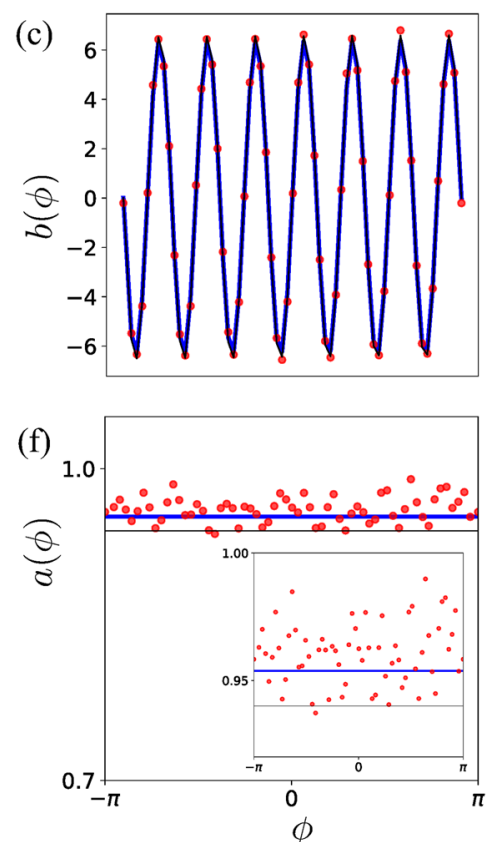

FIG. 7. Sparse regression results for both drift $b$ (upper panels) and diffusion $a$ (lower panels) are summarized, using the same notation and color code as in Fig. 3. [Panels (a) and (d)] Cross validation score $\delta$ as a function of the solution size $n$, with vertical red lines indicating the optimal solutions $\tilde{n}_{b}$ and $\tilde{n}_{a}$. [Panels (b) and (e)] Sparsity progress matrices, where entries associated with the analytic functions are highlighted with color. [Panels (c) and (f)] Comparison between actual drift (diffusion) as from Eq. (34) (black line) and the optimal sparse representations $\tilde{b}(\phi)(\tilde{a}(\phi))$ obtained with the SSR algorithm (blue). Red markers are used to indicate estimation from sampling according to Eqs. (12) and (13). 


$$
\begin{gathered}
C_{r}=\int_{0}^{\infty} r^{-1} \exp \left[-10 \beta(r-1)^{2}-\beta / r\right] d r, \\
Z_{r}=\int_{0}^{\infty} r \exp \left[-10 \beta(r-1)^{2}-\beta / r\right] d r, \\
\frac{C_{r}}{Z_{r}}=0.94, \quad \frac{C_{r}}{Z_{r}} \kappa=6.61 .
\end{gathered}
$$

Both the effective drift $b(\phi)$ and diffusion term $a(\phi)$ are shown in Figs. 7(c) and 7(f) as a reference, as black lines.

A stochastic trajectory was generated by simulating a diffusion process in the two-dimensional potential Eq. (33), and the binned averages of Eqs. (15) and (16) were computed from the simulation data, see Appendix A 1. The time increment $s$ was chosen to be equal to the integration step. A dictionary $\Theta^{\prime \prime}$ of $K=20$ basis functions was used, with composition given in Appendix A, Eq. (A4). The dictionary includes the analytic functions for both $b$ and $a,[1, \sin 7 \phi]$, Eq. (34), to which we refer as analytic basis functions, as in Sec. IV.

The SSR algorithm with cross validation was used to obtain a sparse expression for the estimated drift and diffusion terms. The results are summarized in Fig. 7, where the same notation as in Fig. 4 is used.

The cross validation score plots in Figs. 7(a) and 7(d) allow us to locate the $\delta$ transition points and identify the optimal solutions to Eqs. (17) and (18).
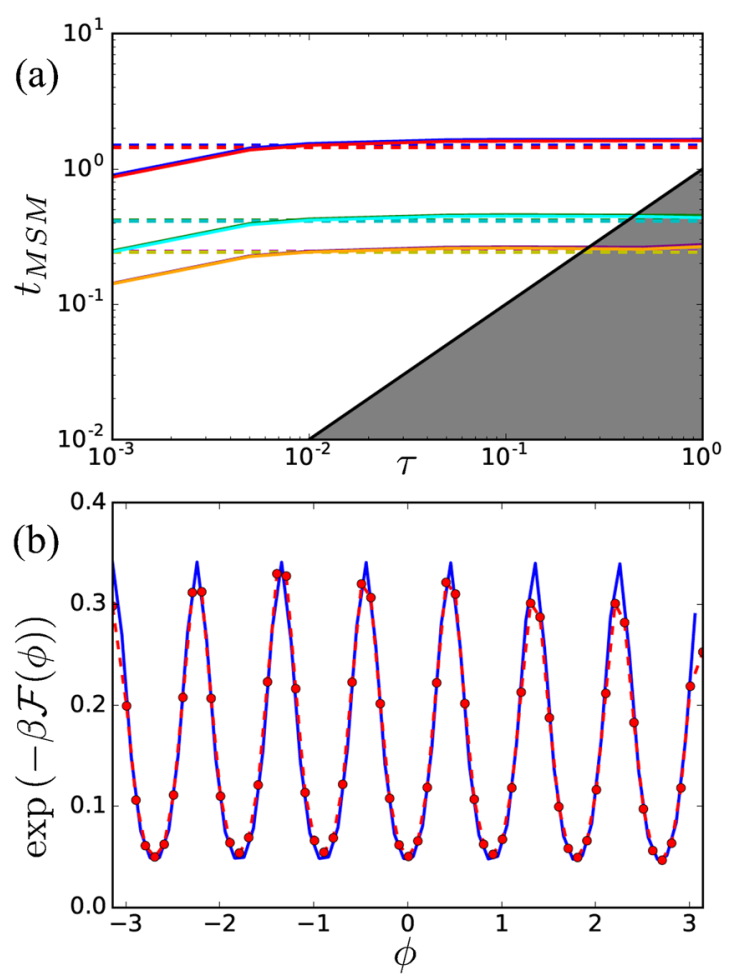

FIG. 8. Comparison between the kinetics and thermodynamics from both the analytic and the data-learned $\phi$ projected diffusion. (Top panel) MSM implied time scales plotted as function of the lag time $\tau$ : horizontal dashed lines indicate the actual time scales of the full system. Deviations between the two dynamics (solid lines) are minimal. (Bottom panel) The stationary distributions $\exp (-\beta \mathcal{F}(\phi))$ of both the analytic (blue solid line) and the reconstructed dynamics $(\tilde{b}, \tilde{a})$ (dashed line with markers indicating the bin centers) are compared: oscillation frequency is matched exactly, and there is a mimimal deviation in the amplitudes because of sampling errors, particularly close to the top of the oscillation.
As $\delta[n]$ reaches its minimum at solution size $n=1$ for both the drift and the diffusion, looking at the $\delta[n-1] / \delta[n]$ ratio is hereby unnecessary.

The optimal sparsity values $\tilde{n}_{b}=1$ and $\tilde{n}_{a}=1$ are indicated by vertical red lines. The optimal solutions read as

$$
\begin{aligned}
\tilde{b}(\phi) & =\tilde{c}_{b} \cdot \Theta(\phi)=6.39 \sin (7 \phi), & & \delta=4.0 \cdot 10^{-2}, \\
\tilde{a}(\phi) & =\tilde{c}_{a} \cdot \Theta(\phi)=0.95, & & \delta=2.0 \cdot 10^{-4},
\end{aligned}
$$

as shown in the sparsity progress matrices in Figs. 7(b) and $7(\mathrm{e})$.

Enforcing sparsity isolates the analytic functions in the dictionary and makes the solutions of Eq. (37) analogous to Eq. (34), as shown in the comparison plots of Figs. 7(c) and 7(f).

We simulated the "learned" projected dynamics $d \phi_{t}$ $=\tilde{b}(\phi) d t+\sqrt{2 \tilde{a}(\phi) \beta^{-1}} d W_{t}$ using the same simulation parameters as in the original dynamics [Eq. (8) with the potential of Eq. (33)]. We discretized the trajectory data and performed a Markov State Model analysis. ${ }^{29}$ Figure 8(a) shows that the first few time scales of the original dynamics (dashed lines) are accurately recovered by the learned projected dynamics (solid lines). Also, the $\phi$-projected equilibrium distributions of both dynamics agree, as shown in Fig. 8(b). Thus, the learned projected dynamics recovers both the full thermodynamics and long time scale kinetics of the original dynamics.

\section{DISCUSSION}

The CV-based sparse regression method SSR presented above appears to be effective at learning stochastic dynamical equations. Indeed, the CV analysis allows us to identify the optimal solution as sparsity is maximized while the model predictive power is preserved. The key features of the drift and the diffusion components (e.g., the dynamics) are shown to be preserved.

For the one dimensional potential Eq. (26), the optimal solution closely approximates the gradient of the potential, as shown in Figs. 3(d) and 4(d). Similarly, the optimal sparse solution for the lemon slice projected dynamics reproduces the thermodynamics and the long time scale kinetics of the original model [see Figs. 7(c), 7(f), and 8].

However, the performance of the SSR algorithm and the specific form of the optimally sparse solution both depend on the composition of the dictionary of basis functions used [e.g., compare $\tilde{\mathbf{c}}$ in Fig. 3(b) with $\tilde{\mathbf{c}}^{\prime}$ in Fig. 4(b)]. The "correct" solution $\tilde{\theta}=\left[1, x, x^{2}, x^{3}\right]$ can be obtained if the solution space is searched greedily, but the SSR algorithm may return solutions corresponding to local minima and multiple function dictionaries need to be considered.

Using ill-conditioned dictionaries containing collinear basis functions exacerbates the convergence problem as multiple linear combinations are almost equivalent, and picking one over others is driven by small perturbations due to numerical noise.

Building a dictionary of strictly linearly independent basis functions (such as Hermite polynomials or Fourier series) is a well-established strategy to avoid the "many-solution" problem from the very beginning. Another popular approach is to run singular value decomposition (SVD) on the regressor 
matrix and discard these singular values which are within machine precision (and therefore contribute exclusively to noise). The reduced set of truncated singular values naturally defines a space into which the regressor matrix can be projected. The regression can then be formulated in terms of new effective variables that are linear combinations of the original ones and are less noisy by construction; hence, more numerical stability is guaranteed. However, if the goal is learning a sparse representation of the potential energy driving a dynamical system, dictionary entries bear a physical meaning, and such interpretation may be lost upon SVD or orthogonalization. For instance, if the input coordinates are composed by a set of contacts or coordinates or angles in a macromolecule, linear combinations of such quantities may be far from being physically interpretable.

The main problem in the convergence of the SSR algorithm is that functions are pruned from the dictionary at every iteration in a deterministic fashion: whenever a given entry is removed from the database, it cannot "resuscitate" in the next iterations, and the solution may be funneled into a local minimum. We believe that introducing stochasticity in the pruning of entries and allowing the reintroduction of previously eliminated entries, resembling Monte Carlo techniques, could significantly improve the performance of the algorithm and also compensate (at least partially) for collinearity.

Additionally, the approach we adopted to numerically estimate the drift and diffusion off trajectory data could easily be replaced by an alternative strategy if needed, as long as it leads to a regression problem: eventually, sparsity is still enforced in the same fashion. The increment method we propose Eqs. (9) and (10) has the advantage of being parameterfree, compared for instance to the work by Stock et al., ${ }^{30,31}$ where for each trajectory point a number of neighbors have to be defined.

The extension of this approach to real high-dimensional molecular systems may be non-trivial. The main issue which could limit the method's applicability as is is that coordinate binning (used here to reduce the effect of the noise) is not a viable approach in high dimension. Thus, the time derivative and the dictionary of basis functions $\Theta$ have to be computed at each trajectory point $X\left(t_{l}\right), l=1, \ldots, L$, leading to large non-sparse matrices whose computation and storage could be computationally challenging. Moreover, it is reasonable to expect that a dictionary of multivariate basis functions is more prone to redundancy, with a large number of suboptimal minima. Current studies address these issues directly.

\section{ACKNOWLEDGMENTS}

We are indebted to Frank Noé, Ralf Banisch, Stefan Klus, Péter Koltai, and Steve Brunton for fruitful discussions. This work was supported by the National Science Foundation (Nos. CHE-1265929, CHE-1738990, and PHY1427654) and the Welch Foundation (No. C-1570). F.N. is a postdoctoral researcher in the Rice University Academy of Fellows. Simulations have been performed on the computer clusters of the Center for Research Computing at Rice University, supported in part by the Big-Data Private-Cloud
Research Cyberinfrastructure MRI-award (NSF Grant No. CNS-1338099).

\section{APPENDIX A: DETAILS}

\section{Binning}

Solving sparse regression Eq. (22) usually involves computing, storing, and inverting large matrices (e.g., $\mathbb{X}, \mathbb{Y}$ ) which scale linearly with the number of frames in a trajectory $N \gg 1$. If the dimension of the system is relatively small, e.g., $d \approx 1$, the problem can be made more tractable numerically. Let us start by histogramming the coordinate $X$ into $Q$ bins, i.e.,

$$
\left\{X\left(t_{l}\right)\right\}_{l=1, \ldots, N} \mapsto\left\{\bar{x}_{i}, w_{i}\right\}_{i=1, \ldots, Q},
$$

where $\bar{x}_{i}$ indicates the $i$ th bin center and $w_{i}$ indicates the fraction of data in the $i$ th bin, which we call bin weight. Subsequently,

$$
\begin{gathered}
\mathbb{X} \in \mathbb{R}^{N \times K} \mapsto \mathbb{X}_{Q} \in \mathbb{R}^{Q \times K} \\
\mathbb{Y} \in \mathbb{R}^{N} \mapsto \mathbb{Y}_{Q} \in \mathbb{R}^{Q}
\end{gathered}
$$

where $Y_{Q}$ entries are averaged over each bin.

The sparse regression Eq. (22) can be cast in the following weighted regression:

$$
\tilde{\mathbf{c}}=\underset{\mathbf{c}}{\arg \min }\left\|\mathbb{W}_{Q} \mathbb{Y}_{Q}-\mathbb{W}_{Q} \mathbb{X}_{Q} \mathbf{c}\right\|_{2}^{2}+\lambda\|\mathbf{c}\|_{0},
$$

where the weight matrix $\mathbb{W}_{Q}$ is defined as

$$
\mathbb{X}_{Q}=\operatorname{diag}\left(w_{1}, \ldots, w_{Q}\right)
$$

Both the double well potential and projected dynamics study cases discussed here are $d=1$ problems, and the binning is used in the results presented in the manuscript.

\section{Double well potential}

The data set $\mathcal{D}$ used in the double well potential example consists of five long independent trajectories of $N=10^{7}$ steps each, generated by integrating the dynamics Eq. (8) using a time increment $s=5 \cdot 10^{-3}$ with $m=1, k_{B} T=1$, and $\gamma=1$ (arbitrary units). The simulations were sufficiently long to ensure sampling from the equilibrium distribution $\pi(x) \propto \exp (-U(x))$, see Eq. (26).

Both the time sequence $\left\{X\left(t_{l}\right)\right\}_{l=1, \cdots N+1}$ and the set of simple increments, Eq. (15),

$$
\left\{Y_{l}\right\}_{l=1, \ldots, N}=\left\{\frac{X\left(t_{l+1}\right)-X\left(t_{l}\right)}{s}\right\}_{l=1, \ldots, N}
$$

were discretized into $Q=90$ bins along the $x$ axis, giving rise to an increment matrix $\mathbb{Y} \in \mathbb{R}^{Q}$.

Two different $K=20$ basis function dictionaries $\Theta$ and $\Theta^{\prime}$ were considered. Both contain the four functions [1, $x$, $x^{2}, x^{3}$ ] entering Eq. (26), the remaining entries were selected randomly from a larger set of 100 basis functions. The specific composition of the two dictionaries reads as 


$$
\begin{aligned}
& \Theta(x)=\left[1, \quad x, \quad x^{2}, \quad x^{3}, \quad x^{4}, \quad x^{5}, \quad x^{6}, \quad x^{7}, \quad x^{8}, \quad x^{9}, \quad x^{10}, \quad \sin x, \quad \cos x,\right. \\
& \left.\sin (6 x), \quad \cos (6 x), \quad \sin (11 x), \quad \cos (11 x), \quad \tanh (10 x), \quad-10 \tanh ^{2}(10 x)+10 e^{-50 x^{2}}\right], \\
& \Theta^{\prime}(x)=\left[1, \quad x, \quad x^{2}, \quad x^{3}, \quad \sin x, \quad \cos 11 x, \quad \sin 11 x, \quad-10 \tanh ^{2}(10 x)+10, \quad-10 \tanh ^{2}(10 x-10)+10,\right. \\
& e^{-50 x^{2}}, \quad e^{-50(x-3)^{2}}, \quad e^{-0.3 x^{2}}, \quad e^{-0.3(x-3)^{2}}, \quad e^{-2(x-2)^{2}}, \quad e^{-2(x-4)^{2}}, \quad e^{-50(x-4)^{2}}, \quad e^{-0.6(x-4)^{2}}, \quad e^{-0.6(x-3)^{2}}, \\
& \left.-2 \tanh ^{2}(2 x-4)+2, \quad \tanh ^{2}(x-4)+1\right] .
\end{aligned}
$$

Each dictionary computed on the binned coordinate generates a database $\mathbb{X} \in \mathbb{R}^{Q \times K}: X_{i j}=\Theta_{i}\left(\bar{x}_{j}\right)$, with $\bar{x}_{j}$ being the value of the coordinate in the $j$ th bin, Eq. (A1).

$N_{k}=50$ independent cross validation calculations were run and the cross validation scores averaged

$$
\delta^{2}=\frac{1}{N_{k}} \sum_{k=1}^{N_{k}} \delta_{k}^{2}
$$

where $\delta_{k}$ is given by Eq. (24). Each CV run, $i$, is associated with its own decomposition of the data set into folds

$$
\mathcal{D} \rightarrow\left\{A_{i}^{j}\right\}_{j=1, \ldots, 5}
$$

\section{Greedy sparsity search}

We provide a short description of the parameters used in the greedy search in the solution space for the double well potential.

The number of independent dictionary combinations reads as

$$
m_{n}=\left\{\begin{array}{ll}
C_{M, n} & \text { if } n \in[2,3,4] \\
10^{5} & \text { otherwise }
\end{array} .\right.
$$

Cross validation scores were computed by running $N_{k}=20$ independent 5-fold cross validations and averaging over all runs as already mentioned.

\section{Projected dynamics}

The dataset $\mathcal{D}$ for the lemon slice example consists of one single $N=10^{7}$ step diffusive trajectory, generated by integrating dynamics Eq. (8) for $U=U(x, y)$ using a time increment $s=10^{-3}$ with $m=1, k_{B} T=1$, and $\gamma=1$ (arbitrary units). The simulation was long enough to ensure equilibrium sampling. The projection coordinate is the polar angle $\xi=\phi$.

Both the time sequence $\left\{\phi\left(x\left(t_{l}\right), y\left(t_{l}\right)\right)\right\}_{l=1, \ldots, N+1}$, the simple increments, Eq. (15),

$$
\left\{Y_{b}(\phi)\right\}_{l=1, \ldots, N}=\left\{\frac{\phi\left(t_{l+1}\right)-\phi\left(t_{l}\right)}{s}\right\}_{l=1, \ldots, N},
$$

and the squared increments, Eq. (16),

$$
\left\{Y_{a}(\phi)\right\}_{l=1, \ldots, N}=\left\{\frac{1}{2} \frac{\left(\phi\left(t_{l+1}\right)-\phi\left(t_{l}\right)\right)^{2}}{s}\right\}_{l=1, \ldots, N}
$$

were discretized into 63 bins along the $\phi \in[-\pi, \pi]$ axis, giving rise to $\mathbb{Y}_{a}, \mathbb{Y}_{b} \in \mathbb{R}^{Q}$.

A $K=20$ basis function dictionary $\Theta^{\prime \prime}$ was considered such that $[1, \sin 7 x] \in \Theta^{\prime \prime}$, Eq. (34). Its specific composition reads as

$$
\begin{aligned}
& \Theta^{\prime \prime}(x)=\left[1, \quad x, \quad x^{2}, \quad x^{3}, \quad x^{4}, \quad x^{5}, \quad x^{6}, \quad \sin x, \quad \cos x \quad \sin 4 x, \quad \cos 4 x, \quad \sin 7 x, \quad \cos (7 x), \quad \tanh (10 x),\right. \\
& \left.-10 \tanh ^{2}(10 x)+10, \quad e^{-50 x^{2}}, \quad \tanh (2 x), \quad-2 \tanh ^{2}(2 x)+2, \quad e^{-2 x^{2}}, \quad \tanh (x)\right] .
\end{aligned}
$$

The functions in the dictionary computed on the binned coordinate generate a database $\mathbb{X} \in \mathbb{R}^{Q \times K}: X_{i j}=\Theta_{i}^{\prime \prime}\left(\bar{\phi}_{j}\right)$, with $\bar{\phi}_{j}$ being the value of the coordinate in the $j$ th angular bin [notation from Eq. (A1)].

Cross validation scores were computed by running $N_{k}=50$ independent 7-fold cross validations and averaging over all runs as already mentioned.

\section{APPENDIX B: PROOF OF THEOREM 1}

We start by collecting a few facts about the projected dynamics: for functions $f, g$ of the full state space $\mathbb{R}^{d}$, the $L^{2}$ scalar product with respect to the measure $\mu$ is defined by

$$
\langle f, g\rangle_{\mu}=\int_{\mathbb{R}^{d}} f(x) g(x) \mathrm{d} \mu(x),
$$

while for functions $u, v$ of the projected space $\mathbb{R}^{m}$, the $L^{2}$ scalar product with respect to the measure $v$ is analogously defined by

$$
\langle u, v\rangle_{v}=\int_{\mathbb{R}^{m}} u(z) v(z) \mathrm{d} v(z) .
$$

The projection operator $\mathcal{P}$ maps a function $f(x)$ on $\mathbb{R}^{d}$ to $\mathcal{P} f(z)$ on $\mathbb{R}^{m}$ by averaging the function over level sets $\Sigma_{z}$

$$
\mathcal{P} f(z)=\int_{\Sigma_{z}} f(x) \mathrm{d} \mu_{z}(x) .
$$


For functions $f(x) \in L_{\mu}^{2}, u(z) \in L_{\nu}^{2}$, the following identity can be verified: ${ }^{11}$

$$
\int_{\mathbb{R}^{d}} f(x) u(\xi(x)) \mathrm{d} \mu(x)=\int_{\mathbb{R}^{m}} \mathcal{P} f(z) u(z) \mathrm{d} v(z) .
$$

As a special case, the last equality yields the identity of the two scalar products for function in $L_{v}^{2}$

$$
\int_{\mathbb{R}^{d}} u(\xi(x)) v(\xi(x)) \mathrm{d} \mu(x)=\int_{\mathbb{R}^{m}} u(z) v(z) \mathrm{d} v(z) .
$$

Now, regression problems, Eqs. (17) and (18), are equivalent to the normal equations

$$
\begin{aligned}
\Theta^{T} \Theta c_{i} & =\Theta^{T} \mathbb{Y}_{i} \\
\Theta^{T} \Theta c_{i j} & =\Theta^{T} \mathbb{Z}_{i j}
\end{aligned}
$$

By ergodicity of the process, for almost surely all trajectories, we have that

$$
\begin{aligned}
\frac{1}{L}\left[\Theta^{T} \Theta\right]_{k, k^{\prime}} & =\frac{1}{L} \sum_{l=1}^{L} f_{k}\left(X\left(t_{l}\right)\right) f_{k^{\prime}}\left(X\left(t_{l}\right)\right) \\
\stackrel{L \rightarrow \infty}{\longrightarrow} \int_{\mathbb{R}^{d}} f_{k}(\xi(x)) f_{k^{\prime}}(\xi(x)) \mathrm{d} \mu(x), & \\
\frac{1}{L}\left[\Theta^{T} \mathbb{Y}_{i}\right]_{k} & =\frac{1}{L} \sum_{l=1}^{L} f_{k}\left(X\left(t_{l}\right)\right) \frac{1}{s} e_{i}^{\xi}\left(X\left(t_{l+1}\right), X\left(t_{l}\right)\right) \\
\stackrel{L \rightarrow \infty}{\longrightarrow} & \int_{\mathbb{R}^{d}} f_{k}(\xi(x)) \mathbb{E}^{x}\left[\frac{1}{s} e_{i}^{\xi}(X(s), X(0))\right] \mathrm{d} \mu(x), \\
\frac{1}{L}\left[\Theta^{T} \mathbb{Z}_{i j}\right]_{k} & \frac{\beta}{2} \frac{1}{L} \sum_{l=1}^{L} f_{k}\left(X\left(t_{l}\right)\right) \frac{1}{s} e_{i}^{\xi}\left(X\left(t_{l+1}\right), X\left(t_{l}\right)\right) e_{j}^{\xi}\left(X\left(t_{l+1}\right), X\left(t_{l}\right)\right) \\
\stackrel{L \rightarrow \infty}{\longrightarrow} & \frac{\beta}{2} \int_{\mathbb{R}^{d}} f_{k}(\xi(x)) \mathbb{E}^{x}\left[\frac{1}{s} e_{i}^{\xi}(X(s), X(0)) e_{j}^{\xi}(X(s), X(0))\right] \mathrm{d} \mu(x) .
\end{aligned}
$$

By Eqs. (B4) and (B5), these expressions can be transformed into

$$
\begin{aligned}
& \frac{1}{L}\left[\Theta^{T} \Theta\right]_{k, k^{\prime}} \stackrel{L \rightarrow \infty}{\longrightarrow} \int_{\mathbb{R}^{m}} f_{k}(z) f_{k^{\prime}}(z) \mathrm{d} v(z), \\
& \frac{1}{L}\left[\Theta^{T} \mathbb{Y}_{i}\right]_{k} \stackrel{L \rightarrow \infty}{\longrightarrow} \int_{\mathbb{R}^{m}} f_{k}(z) \mathcal{P}\left[\mathbb{E}^{x}\left[\frac{1}{s} e_{i}^{\xi}(X(s), X(0))\right]\right] \mathrm{d} v(z), \\
&=\int_{\mathbb{R}^{m}} f_{k}(z) \mathbb{E}\left[\frac{1}{s} e_{i}^{\xi}\left(X(s), z_{i}\right) \mid X_{0} \sim \mu_{z}\right] \mathrm{d} v(z), \\
& \frac{1}{L}\left[\Theta^{T} \mathbb{Z}_{i j}\right]_{k} \stackrel{L \rightarrow \infty}{\longrightarrow} \frac{\beta}{2} \int_{\mathbb{R}^{m}} f_{k}(z) \mathbb{E}\left[\frac{1}{s} e_{i}^{\xi}\left(X(s), z_{i}\right) e_{j}^{\xi}\left(X(s), z_{j}\right) \mid X_{0} \sim \mu_{z}\right] \mathrm{d} v(z) .
\end{aligned}
$$

In the limit $s \rightarrow 0$, the Kramers-Moyal formulae imply that

$$
\begin{array}{r}
\int_{\mathbb{R}^{m}} f_{k}(z) \mathbb{E}\left[\frac{1}{s} e_{i}^{\xi}\left(X(s), z_{i}\right) \mid X_{0} \sim \mu_{z}\right] \mathrm{d} v(z) \stackrel{s \rightarrow 0}{\longrightarrow} \int_{\mathbb{R}^{m}} f_{k}(z) b_{i}^{\xi}(z) \mathrm{d} v(z), \\
\frac{\beta}{2} \int_{\mathbb{R}^{m}} f_{k}(z) \mathbb{E}\left[\frac{1}{s} e_{i}^{\xi}\left(X(s), z_{i}\right) e_{j}^{\xi}\left(X(s), z_{j}\right) \mid X_{0} \sim \mu_{z}\right] \mathrm{d} v(z) \stackrel{s \rightarrow 0}{\longrightarrow} \int_{\mathbb{R}^{m}} f_{k}(z) a_{i j}^{\xi}(z) \mathrm{d} v(z),
\end{array}
$$

showing that the solutions of the normal equations, Eqs. (B6) and (B7), converge to the solutions of the best approximation problems for $b^{\xi}$ and $a^{\xi}$ in the space $L_{v}^{2}$.

\section{APPENDIX C: ESTIMATION OF POTENTIAL ENERGY}

The regression problem Eq. (21) also converges to the best-approximation problem for the generalized energy Eq. (19) from the basis set of vector fields $\nabla f_{k}, k=1, \ldots, K$. The case where no projection is applied can be recovered by choosing $\xi$ as the identity on $\mathbb{R}^{n}$. The proof uses the same arguments as in Appendix B, the only difference is the summation over all degrees of freedom. Starting from the normal equations

$$
\mathbb{D}^{T} \mathbb{D} v=\mathbb{D}^{T} \mathbb{Y},
$$

we first find that

$$
\begin{aligned}
& \frac{1}{L}\left[\mathbb{D}^{T} \mathbb{D}\right]_{k, k^{\prime}}=\frac{1}{L} \sum_{i, l} \frac{\partial f_{k}}{\partial z_{i}}\left(X\left(t_{l}\right)\right) \frac{\partial f_{k^{\prime}}}{\partial z_{i}}\left(X\left(t_{l}\right)\right) \\
& \stackrel{L \rightarrow \infty}{\longrightarrow} \sum_{i=1}^{m} \int_{\mathbb{R}^{d}} \frac{\partial f_{k}(\xi(x))}{\partial z_{i}} \frac{\partial f_{k^{\prime}}(\xi(x))}{\partial z_{i}} \mathrm{~d} \mu(x) \\
&=\int_{\mathbb{R}^{m}}\left\langle\nabla f_{k}, \nabla f_{k^{\prime}}\right\rangle \mathrm{d} v(z) .
\end{aligned}
$$


Moreover, the data vector $D^{T} Y$ converges to

$$
\begin{aligned}
\frac{1}{L}\left[\mathbb{D}^{T} \mathbb{Y}\right]_{k} & =\frac{1}{L} \sum_{i, l} \frac{\partial f_{k}}{\partial z_{i}}\left(X\left(t_{l}\right)\right)\left[\left(a^{\xi}\right)^{-1}\left(X\left(t_{l}\right)\right)\left(\frac{1}{\beta} \nabla \cdot a_{i}^{\xi}\left(X\left(t_{l}\right)\right)-\frac{1}{s} e^{\xi}\left(X\left(t_{l+1}\right), X\left(t_{l}\right)\right)\right)\right]_{i} \\
& \stackrel{L \rightarrow \infty}{\longrightarrow} \sum_{i=1}^{m} \int_{\mathbb{R}^{d}} \frac{\partial f_{k}(\xi(x))}{\partial z_{i}}\left[\left(a^{\xi}\right)^{-1}(\xi(x))\left(\frac{1}{\beta} \nabla \cdot a_{i}^{\xi}(\xi(x))-\mathbb{E}^{x}\left[\frac{1}{s} e^{\xi}(X(s), X(0))\right]\right)\right]_{i} \mathrm{~d} \mu(x) \\
& =\sum_{i=1}^{m} \int_{\mathbb{R}^{m}} \frac{\partial f_{k}(z)}{\partial z_{i}}\left[\left(a^{\xi}\right)^{-1}(z)\left(\frac{1}{\beta} \nabla \cdot a_{i}^{\xi}(z)-\mathbb{E}\left[\frac{1}{s} e^{\xi}(X(s), z) \mid X(0) \sim \mu_{z}\right]\right)\right]_{i} \mathrm{~d} v(z) \\
& \stackrel{s \rightarrow 0}{\longrightarrow} \sum_{i=1}^{M} \int_{\mathbb{R}^{m}} \frac{\partial f_{k}(z)}{\partial z_{i}}\left[\left(a^{\xi}\right)^{-1}\left(\frac{1}{\beta} \nabla \cdot a_{i}^{\xi}-b^{\xi}\right)\right]_{i}(z) \mathrm{d} v(z) \\
& =\int_{\mathbb{R}^{m}}\left\langle\nabla f_{k}(z), \nabla \mathcal{F}(z)\right\rangle \mathrm{d} v(z) .
\end{aligned}
$$

${ }^{1}$ F. Noé and C. Clementi, Curr. Opin. Struct. Biol. 43, 141 (2017).

${ }^{2}$ M. A. Rohrdanz, W. Zheng, and C. Clementi, Annu. Rev. Phys. Chem. 64, 295 (2013).

${ }^{3}$ I. G. Kevrekidis and G. Samaey, Annu. Rev. Phys. Chem. 60, 321 (2009).

${ }^{4}$ S. L. Brunton, J. L. Proctor, and J. N. Kutz, Proc. Natl. Acad. Sci. U. S. A. 113, 3932 (2016).

${ }^{5}$ R. Tibshirani, J. R. Stat. Soc. B 58, 267 (1996).

${ }^{6}$ G. James, D. Witten, T. Hastie, and R. Tibshirani, An Introduction to Statistical Learning (Springer New York, 2013).

${ }^{7}$ D. Donoho, IEEE Trans. Inf. Theory 52, 1289 (2006).

${ }^{8}$ R. Baraniuk, IEEE Signal Process. Mag. 24, 118 (2007).

${ }^{9}$ S. H. Rudy, S. L. Brunton, J. L. Proctor, and J. N. Kutz, Sci. Adv. 3, e1602614 (2017).

${ }^{10} \mathrm{~F}$. Legoll and T. Lelièvre, Nonlinearity 23, 2131 (2010).

${ }^{11}$ W. Zhang, C. Hartmann, and C. Schütte, Faraday Discuss. 195, 365 (2016).

${ }^{12} \mathrm{H}$. Risken and H. Haken, The Fokker-Planck Equation: Methods of Solution and Applications, 2nd ed. (Springer, 1989).

${ }^{13}$ M. Kessler, A. Lindner, and M. Sorensen, Statistical Methods for Stochastic Differential Equations (CRC Press, 2012).

${ }^{14}$ L. Zhang, P. A. Mykland, and Y. Aitt-Sahalia, J. Am. Stat. Assoc. 100, 1394 (2005).

${ }^{15}$ E. Gobet, M. Hoffmann, M. Reiß et al., Ann. Stat. 32, 2223 (2004).

${ }^{16}$ D. Crommelin and E. Vanden-Eijnden, Multiscale Model. Simul. 9, 1588 (2011).

${ }^{17}$ G. A. Pavliotis and A. Stuart, J. Stat. Phys. 127, 741 (2007).

${ }^{18}$ S. Kalliadasis, S. Krumscheid, and G. A. Pavliotis, J. Comput. Phys. 296, 314 (2015)
${ }^{19}$ G. Pavliotis, Stochastic Processes and Applications: Diffusion Processes, the Fokker-Planck and Langevin Equations, Texts in Applied Mathematics (Springer New York, 2014).

${ }^{20}$ R. Tibshirani, J. R. Stat. Soc. B 73, 273 (2011).

${ }^{21}$ S. G. Mallat and Z. Zhang, IEEE Trans. Signal Process. 41, 3397 (1993).

${ }^{22}$ Y. C. Pati, R. Rezaiifar, and P. S. Krishnaprasad, in Proceedings of 27th Asilomar Conference on Signals, Systems and Computers (IEEE, 1993), pp. 40-44.

${ }^{23}$ H. Zou and T. Hastie, J. R. Stat. Soc. B 67, 301 (2005).

${ }^{24}$ T. Hastie, R. Tibshirani, and J. Friedman, The Elements of Statistical Learning, Springer Series in Statistics (Springer New York, Inc., New York, NY, USA, 2001).

${ }^{25} \mathrm{R}$. Kohavi, in Proceedings of the 14th International Joint Conference on Artificial Intelligence-Volume 2, IJCAI'95 (Morgan Kaufmann Publishers, Inc., San Francisco, CA, USA, 1995), pp. 1137-1143.

${ }^{26}$ S. Geisser, J. Am. Stat. Assoc. 70, 320 (1975).

${ }^{27}$ F. Pedregosa, G. Varoquaux, A. Gramfort, V. Michel, B. Thirion, O. Grisel, M. Blondel, P. Prettenhofer, R. Weiss, V. Dubourg, J. Vanderplas, A. Passos, D. Cournapeau, M. Brucher, M. Perrot, and E. Duchesnay, J. Mach. Learn. Res. 12, 2825 (2011).

${ }^{28}$ A. Bittracher, P. Koltai, S. Klus, R. Banisch, M. Dellnitz, and C. Schütte, J. Nonlinear Sci. 28, 471 (2017).

${ }^{29}$ J.-H. Prinz, H. Wu, M. Sarich, B. Keller, M. Senne, M. Held, J. D. Chodera, C. Schütte, and F. Noé, J. Chem. Phys. 134, 174105 (2011).

${ }^{30}$ R. Hegger and G. Stock, J. Chem. Phys. 130, 034106 (2009).

${ }^{31}$ N. Schaudinnus, A. J. Rzepiela, R. Hegger, and G. Stock, J. Chem. Phys. 138, 204106 (2013). 